\title{
GREEN'S FUNCTION POINTWISE ESTIMATES FOR THE MODIFIED LAX-FRIEDRICHS SCHEME
}

\author{
PAUline Godillon ${ }^{1}$
}

\begin{abstract}
The aim of this paper is to find estimates of the Green's function of stationary discrete shock profiles and discrete boundary layers of the modified Lax-Friedrichs numerical scheme, by using techniques developed by Zumbrun and Howard [27] in the continuous viscous setting.
\end{abstract}

Mathematics Subject Classification. 35L65.

Received: March 7, 2002.

\section{INTRODUCTION}

We deal at first with the case of discrete shock profiles: let $d \geq 1$ and consider the one-dimensional $d \times d$ system of conservation laws

$$
\begin{gathered}
u_{t}+f(u)_{x}=0, t \geq 0, \quad x \in \mathbb{R}, \\
u: \mathbb{R} \times \mathbb{R}^{+} \longrightarrow \mathcal{U}, \mathcal{U} \text { an open set of } \mathbb{R}^{d}, \\
f: \mathcal{U} \subset \mathbb{R}^{d} \longrightarrow \mathbb{R}^{d} \text { smooth, }
\end{gathered}
$$

with an initial datum

$$
u(x, 0)=\mathrm{u}_{0}(x), x \in \mathbb{R}
$$

We are interested here in the approximation of (1) by means of the modified Lax-Friedrichs scheme (MLF). We consider a uniform mesh of $\mathbb{R}$ consisting of cells $\mathcal{M}_{j}:=(j h,(j+1) h]$ of size $h$ with $j \in \mathbb{Z}$. The time step is $k$ and we set $t^{n}=n k, n \in \mathbb{N}$. Let $\mathcal{N}$ be the (nonlinear) evolution operator associated with the MLF scheme

$$
(\mathcal{N} u)_{j}=u_{j}-\frac{k}{h}\left(\mathcal{F}\left(u_{j}, u_{j+1}\right)-\mathcal{F}\left(u_{j-1}, u_{j}\right)\right), j \in \mathbb{Z},
$$

where the numerical flux $\mathcal{F}$ is

$$
\mathcal{F}(u, v)=\frac{f(u)+f(v)}{2}+\mathbf{D}(u-v)
$$

Keywords and phrases. Linear stability, discrete shock profiles, Laplace transform.

1 Unité de Mathématiques Pures et Appliquées, CNRS UMR \# 5669, ENS Lyon, 46 allée d'Italie, 69364 Lyon Cedex 07, France. e-mail: pgodillo@umpa.ens-lyon.fr 
with $\mathbf{D}$ a scalar constant.

The numerical problem associated with (1)-(3) is

$$
\begin{aligned}
u^{n+1} & =\mathcal{N}\left(u^{n}\right), n \geq 1, \\
u_{j}^{0} & =\frac{1}{\left|\mathcal{M}_{j}\right|} \int_{\mathcal{M}_{j}} \mathrm{u}_{0}(x) \mathrm{d} x, j \in \mathbb{Z} .
\end{aligned}
$$

Let us now make the standard assumptions. Let $\left(u^{-}, u^{+}\right)$be a stationary shock of (1) of arbitrary strength, that satisfies the following hypotheses (H1)-(H5):

- the Rankine-Hugoniot condition is satisfied:

Hypothesis 1. $f\left(u^{+}\right)=f\left(u^{-}\right)$;

- $\operatorname{system}(1)$ is strictly hyperbolic at the points $u^{ \pm}$:

Hypothesis 2. $\mathrm{d} f\left(u^{ \pm}\right)$is diagonalizable and its eigenvalues are real and simple; we denote them by $\mathbf{a}_{1}^{ \pm}<\ldots<\mathbf{a}_{d}^{ \pm}$and by $\left(r_{q}^{ \pm}\right)_{q \in\{1, \ldots, d}$ some associated eigenvectors;

- the shock is non-characteristic:

Hypothesis 3. $0 \notin \sigma\left(\mathrm{d} f\left(u^{ \pm}\right)\right)$, where $\sigma$ denotes the spectrum;

- there are at least $d$ characteristics entering the shock, that is, the eigenvalues of $\mathrm{d} f\left(u^{ \pm}\right)$satisfy the following inequalities:

Hypothesis 4.

$$
\begin{gathered}
\mathbf{a}_{p^{+}}^{+}<0<\mathbf{a}_{p^{-}}^{-}, \\
\mathbf{a}_{p^{-}-1}^{-}<0<\mathbf{a}_{p^{+}+1}^{+},
\end{gathered}
$$

with $p^{+}=p^{-}+\varpi, \varpi \in\{-1, \ldots, d-1\}$.

This last condition implies that there are $d+\varpi+1$ characteristics entering the shock and $d-\varpi-1$ outgoing ones. The case $p^{+}=p^{-}-1$ corresponds to an undercompressive shock [23]. When $p^{+} \geq p^{-}$, the shock is said to be compressive, in reference to the case of gas dynamics $[5,26]$, where the pressure increases through such a shock. More precisely, if $\varpi=0$, the shock is said to be of Lax-type and if $\varpi \geq 1$, the shock is overcompressive of degree $\varpi[16,17]$.

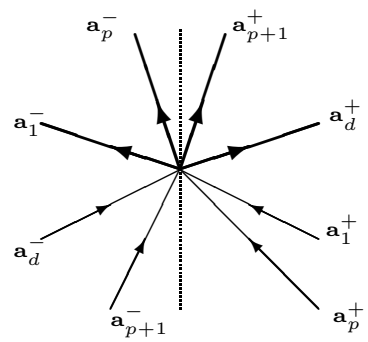

Undercompressive shock $\left(p^{+}=p=p^{-}-1\right)$

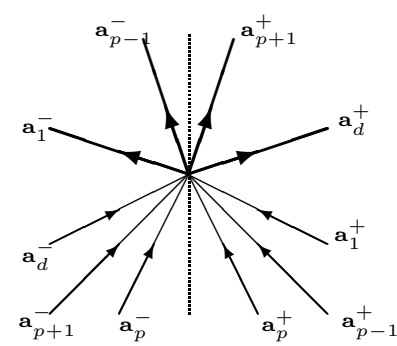

Lax $p$-shock $\left(p^{+}=p^{-}=p\right)$

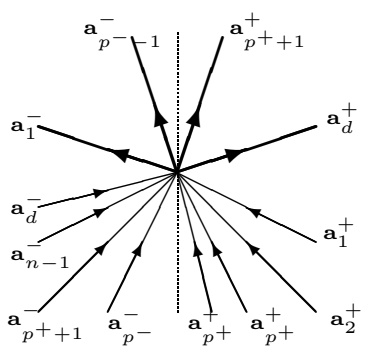

Overcompressive shock $\left(p^{+} \geq p^{-}+1\right)$

Figure 1. Compressivity of the shock.

Our last standard assumption is that the well-known CFL condition is satisfied:

Hypothesis 5. $\sup _{u \in \mathcal{U}} \mathrm{r}(\mathrm{d} f(u))<2 \mathbf{D}<\frac{h}{k}$, where $\mathrm{r}$ denotes the spectral radius. 
We are interested here in stationary discrete shock profiles of the MLF scheme, that is, fixed points of the operator $\mathcal{N}$ that connect the end states $u^{-}$and $u^{+}$. The existence of stationary discrete shock profiles has been studied for quite a long time $[13,20,22]$. When the speed of the shock is not zero, however, the search for discrete shock profiles is much more difficult: in the case of rational speeds, we need to iterate the operator $\mathcal{N}$ to change to a stationary shock, but the iterated operator is much more complicated than the original one; Liu and $\mathrm{Yu}[18,19]$ developed a Diophantine condition under which they proved the existence of discrete shock profiles for weak shocks. The case of irrational speeds remains open [24]. Let us assume now the existence of a stationary discrete shock profile for the MLF scheme:

Hypothesis 6. there exists a sequence $\bar{u}^{\mathrm{s}}=\left(\bar{u}_{j}^{\mathrm{s}}\right)_{j \in \mathbb{Z}}$ that satisfies

$$
\begin{array}{r}
\mathcal{N}\left(\bar{u}^{\mathrm{s}}\right)=\bar{u}^{\mathrm{s}}, \\
\bar{u}_{j}^{\mathrm{s}} \underset{j \rightarrow \pm \infty}{\longrightarrow} u^{ \pm} .
\end{array}
$$

Let us now linearize $\mathcal{N}$ about $\bar{u}^{\text {s }}$

$$
u_{j}^{n+1}=u_{j}^{n}-\left(\frac{F_{j+1} u_{j+1}^{n}-F_{j-1} u_{j-1}^{n}}{2}-\mathbf{D}\left(u_{j+1}^{n}-2 u_{j}^{n}+u_{j-1}^{n}\right)\right)=:\left(L^{\mathrm{s}} u^{n}\right)_{j}, j \in \mathbb{Z},
$$

where

$$
\begin{aligned}
F_{j} & :=\frac{k}{h} \mathrm{~d} f\left(\bar{u}_{j}^{\mathrm{s}}\right), \forall n \in \mathbb{N}, \forall j \in \mathbb{Z}, \\
D & :=\frac{k \mathbf{D}}{h} .
\end{aligned}
$$

In the following, we denote

$$
\begin{aligned}
F^{ \pm} & :=\frac{k}{h} \mathrm{~d} f\left(u^{ \pm}\right), \\
a_{q}^{ \pm} & :=\frac{k}{h} \mathbf{a}_{q}^{ \pm}, q \in\{1, \ldots, d\}
\end{aligned}
$$

Note that $\left(a_{q}^{ \pm}\right)_{q \in\{1, \ldots, n\}}$ are the eigenvalues of $F^{ \pm}$and that $\left(r_{q}^{ \pm}\right)_{q \in\{1, \ldots, n\}}$ are associated eigenvectors.

The numerical linearized problem that we are interested in is

$$
\begin{gathered}
u^{n+1}-L^{\mathrm{s}} u^{n}=\tilde{u}^{n}, \forall n \geq 0, \\
u_{j}^{n} \underset{j \rightarrow \pm \infty}{\longrightarrow} 0, \forall n \geq 0, \\
u^{0}=\mathbf{u},
\end{gathered}
$$

where $\tilde{u}$ and $\mathbf{u}$ are given sequences.

A way to describe the solutions of (9)-(11) is by using the Green's function, that is a sequence $(\mathrm{G}(n, l, j))_{n \in \mathbb{N}, l \in \mathbb{Z}, j \in \mathbb{Z}}$ of $d \times d$ matrices that solves the following problem, $l$ being given in $\mathbb{Z}$,

$$
\begin{aligned}
& \mathrm{G}(n+1, l, j)-L^{\mathrm{s}} \mathrm{G}(n, l, j)=0, \forall n \geq 0, j \in \mathbb{Z}, \\
& \mathrm{G}(n, l, j) \underset{j \rightarrow \pm \infty}{\longrightarrow} 0, \forall n \geq 1, \\
& \mathrm{G}(0, l, j)=\delta_{l j} I_{d}, j \in \mathbb{Z},
\end{aligned}
$$


where $\delta$ is the Kronecker symbol. Indeed, the formal solution of (9)-(11) is given by

$$
u_{j}^{n}=\sum_{l=-\infty}^{+\infty} \mathrm{G}(n, l, j) \mathbf{u}_{l}+\sum_{\bar{n}=0}^{n-1} \sum_{l=-\infty}^{+\infty} \mathrm{G}(n-\bar{n}, l, j) \tilde{u}_{l}^{\bar{n}}, \text { for } j \in \mathbb{Z}, n \geq 1 .
$$

The aim of this paper is to find estimates of $\mathrm{G}$, in order to be able to better understand its behavior, in the same way as Zumbrun and Howard [27] did in the case of (continuous) viscous shock profiles.

The operator $L^{\mathrm{s}}$ is said to be spectrally stable if $L$ has no spectrum outside the open unit disc. A study of the essential spectrum of $L^{\mathrm{s}}$ shows that the spectrum of $L^{\mathrm{s}}$ outside the unit disc consists only of isolated eigenvalues of finite multiplicity. These eigenvalues are unstable modes of $L^{\mathrm{s}}$ [14]. Consequently, to avoid instability, we assume that:

Hypothesis 7. The operator $L^{\mathrm{s}}$ has no eigenvalue of modulus larger or equal to 1 other than 1.

Indeed, in the compressive case $(\varpi \geq 0), 1$ is an eigenvalue of $L^{\mathrm{s}}$ and must be dealt with very carefully (see $[3,24,25])$. An analogous role is played by the eigenvalue 0 in the continuous case: every translation of the shock profile is also a shock profile, so that the derivative of the profile is always an eigenfunction of the linearized operator for the eigenvalue 0 [27]. In the discrete case, however, the translation invariance parameter is an integer and there is no such generality. That is the reason why we must study the operator $L^{\mathrm{s}}-1$ very precisely. To investigate the role of 1 , we use an Evans function, that is a function $\mathcal{D}(j, \mu)$ which is a defined for $|\mu|>1$ by a determinant of $2 d$ solutions of

$$
\left(L^{\mathrm{s}}-\mu\right) v=0 .
$$

The main feature of the Evans function is that it vanishes at points $\mu$ that are eigenvalues of $L^{\mathrm{s}}$. More precisely, considering a basis $v_{1}(\mu, j), \ldots, v_{d}(\mu, j)$ (resp. $\left.v_{j+1}(\mu, j), \ldots, v_{2 d}(\mu, j)\right)$ of the solutions of (15) that decrease exponentially towards 0 as $j$ tends to $+\infty$ (resp. to $-\infty$ ), we define, for $|\mu|>1$ and $j \in \mathbb{Z}$,

$$
\mathcal{D}(\mu, j)=\operatorname{det}\left(\begin{array}{cccccc}
v_{1}(\mu, j) & \ldots & v_{d}(\mu, j) & v_{d+1}(\mu, j) & \ldots & v_{2 d}(\mu, j) \\
\Delta v_{1}(\mu, j) & \ldots & \Delta v_{d}(\mu, j) & \Delta v_{d+1}(\mu, j) & \ldots & \Delta v_{2 d}(\mu, j)
\end{array}\right),
$$

where $\Delta v_{j}=v_{j}-v_{j-1}$, so that $\mathcal{D}$ is holomorphic with respect to $\mu$ in $|\mu|>1$.

In the continuous setting, the Gap Lemma [6] allows to extend the Evans function to a neighborhood of the origin. Thanks to this technique, necessary conditions of spectral stability have been obtained for various approximations, such as Gardner and Zumbrun's [6] and Benzoni, Serre and Zumbrun's [2] for the viscous case, Serre's for the Lax-Friedrichs scheme [25], Bultelle, Grassin and Serre's for the Godunov scheme [3], Benzoni's for the semi-discrete profiles [1], Godillon's for the semi-linear relaxation [10]. Zumbrun and Serre linked, in the multi-dimensional setting, the Evans function and the Lopatinski condition [28].

Here, the study of $\left(v_{q}\right)_{q \in\{1, \ldots, 2 d\}}$ in a neighborhood of $\mu=1$ shows that $\mathcal{D}$ necessarily vanishes at $\mu=1$ if the shock is compressive $(\varpi \geq 0)$. Furthermore, if $\varpi>0$, the $\varpi$ first derivatives of $\mathcal{D}$ with respect to $\mu$ necessarily vanish at $\mu=1$. Consequently, we assume that the shock is minimally degenerated, that is,

Hypothesis 8. $\frac{\partial^{\varpi+1} \mathcal{D}}{\partial \mu^{\varpi+1}}(\cdot, j)$ does not vanish at $\mu=1$ for all $j \in \mathbb{Z}$.

Note that hypothesis (H7) can be reformulated equivalently as:

Hypothesis 7. if $\varpi \geq 0$ (resp. if $\varpi=-1$ ), the Evans function $\mathcal{D}(\mu, \cdot)$ does not vanish in $\{\mu \in \mathbb{C} /|\mu| \geq 1\} \backslash\{1\}$ (resp. $|\mu| \geq 1$ ). 
The aim of this paper is to prove the following theorem:

Theorem 1.1 (Green's function of a shock profile). Assuming (H1)-(H8), the Green's function of the linearized problem (9)-(11) behaves as follows:

$$
\begin{aligned}
\text { for } \vec{e} \in \mathbb{R}^{d}, & \\
\mathrm{G}(n, l, j) \cdot \vec{e}= & \chi_{|j|<n \min \left(\left|a_{q}^{ \pm}\right|, q \in\{1, \ldots, d\}\right)} \mathcal{R}_{0}(l, j) \cdot \vec{e}+\sum_{q / j a_{q}^{ \pm}>0} \frac{1}{\sqrt{n}} O\left(\exp \left(-\frac{\left(j-a_{q}^{ \pm} n\right)^{2}}{M n}\right)\right) r_{q}^{ \pm} \\
& +O\left(\mathrm{e}^{-\gamma n} \exp \left(-\frac{(l-j)^{2}}{M n}\right)\right), l, j \in \mathbb{Z}, \\
\Delta_{j} \mathrm{G}(n, l, j) \cdot \vec{e}= & \chi_{|j|<n \min \left(\left|a_{q}^{ \pm}\right|, q \in\{1, \ldots, d\}\right)} \Delta_{j} \mathcal{R}_{0}(l, j) \cdot \vec{e}+\sum_{q / j a_{q}^{ \pm}>0} \frac{1}{n} O\left(\exp \left(-\frac{\left(j-a_{q}^{ \pm} n\right)^{2}}{M n}\right)\right) r_{q}^{ \pm} \\
& +O\left(\mathrm{e}^{-\gamma n} \exp \left(-\frac{(l-j)^{2}}{M n}\right)\right), l, j \in \mathbb{Z},
\end{aligned}
$$

where

- the notation $\Delta_{j} \mathrm{G}(\cdot, \cdot, j)$ refers to $\mathrm{G}(\cdot, \cdot, j)-\mathrm{G}(\cdot, \cdot, j-1)$;

- the notation $q / j a_{q}^{ \pm}>0$ addresses the indices $q \in\{1, \ldots, d\}$ such that $j$ and the eigenvalues $a_{q}^{\operatorname{sign}(j)}$ have the same sign;

- the residual term $\mathcal{R}_{0}(l, j)$ is a projection on the eigenspace of $L^{\mathrm{s}}$ associated with the eigenvalue 1 (see Eq. (58));

- all the constants are locally bounded on $l$ and uniformly bounded on $n$ and $j$, and the constants $M$ and $\gamma$ are positive.

This result is analogous to the ones proved by Zumbrun and Howard [27] in the case of viscous shock profiles, although they do not set on $y$ (the continuous analogue of our discrete variable $l$ ) to be bounded. At first, the Dirac mass splits into waves that propagate along the outgoing characteristics and waves that propagate along the entering characteristics. The waves that are carried by the outgoing characteristics, that is eigenvectors corresponding to negative (respectively positive) eigenvalues if the Dirac mass was on the left-hand (resp. righthand) side of the shock, take the shape of moving Gaussians that are damped by the numerical viscosity. Their asymptotic speeds are the corresponding eigenvalues of the derivatives of the flux at the end states multiplied by the ratio $k / h$. The waves propagating along the entering characteristics move towards the shock, and when each wave corresponding to a different characteristic reaches the position of the shock $(j=0)$, similar outgoing waves as described above are emitted. Furthermore, if the shock is compressive, as soon as the first entering waves has reached the shock, a stationary residual wave that is strongly related to the kernel of $L^{\mathrm{s}}-1$ may appear, depending on the position of the Dirac mass as initial datum. There is also a fast-time decaying term. Note that the fact that the outgoing waves are Gaussian-shaped is compatible with the $\ell^{1}$ conservation of the mass.

We give in Section 5 a numerical illustration of Theorem 1.1: we treat the case of a Lax 3-shock for the $3 \times 3$ system of gas dynamics in conservative variables. We include graphics displaying the three components of the Green's function in the canonical basis (see Fig. 12) and in the basis of the eigenvectors of $\mathrm{d} f\left(u^{-}\right)$(see Fig. 14), in order to show the different speeds and directions of the waves that appear when the Dirac mass splits. We also compute an eigenfunction of $L^{\mathrm{s}}-1$ (see Figs. 13 and 15) in order to compare it with the residual wave $\mathcal{R}_{0}$. The evolution of the Green's function is compared to the theoretical Gaussians in movies that are available at http://www .umpa.ens-lyon.fr/ pgodillo. 
Let us now give a few hints of the proof of Theorem 1.1. As in the work of Zumbrun and Howard for viscous shock profiles [27], the main tool that we are going to use is the Laplace transform of a sequence that we define by

$$
v=\left(v^{n}\right)_{n \in \mathbb{N}} \mapsto\left(\lambda \in \mathbb{D} \mapsto \hat{v}(\lambda):=\sum_{n \in \mathbb{N}} \mathrm{e}^{-\lambda n} v^{n}\right)
$$

where $\mathbb{D}$ is a subset of $\mathbb{C}$ suitably chosen to ensure the convergence of the sum. In particular, since (16) is $i 2 \pi$ periodic in $\lambda$, we set on $\mathbb{D}$ to lie in the strip $\mathcal{S}:=\{\lambda \in \mathbb{C} /-\pi \leq \operatorname{Im}(\lambda) \leq \pi\}$. Given $l \in \mathbb{Z}$, the Laplace transform of the Green's function $\mathrm{G}(\cdot, l, \cdot)$ with respect to time, that we denote by $\mathrm{G}_{\lambda}(l, \cdot)$, satisfies the following problem

$$
\begin{gathered}
\left(L^{\mathrm{s}}-\mathrm{e}^{\lambda}\right) \mathrm{G}_{\lambda}(l, j)=-\delta_{l j} \mathrm{e}^{\lambda} I_{d}, j \in \mathbb{Z}, \\
\mathrm{G}_{\lambda}(l, j) \underset{j \rightarrow \pm \infty}{\longrightarrow} 0 .
\end{gathered}
$$

In Section 2, rewriting the homogeneous equation

$$
\left(L^{\mathrm{s}}-\mathrm{e}^{\lambda}\right) v=0
$$

as a first-order dynamical system

$$
V_{j}=\mathbb{A}_{j}(\lambda) V_{j-1}, V_{j}=\left(\begin{array}{c}
v_{j} \\
v_{j+1}-v_{j}
\end{array}\right) \in \mathbb{C}^{2 d}, j \in \mathbb{Z},
$$

we study the limit systems of (19) as $j$ tends to $\pm \infty$, and more specifically the behavior of the solutions decreasing to 0 at $\pm \infty$ : it allows us to construct the Evans function $\mathcal{D}(\lambda, j)$, (we take hereafter the variable $\lambda$, that is linked to the variable $\mu$, that we used in (15), through $\mathrm{e}^{\lambda}=\mu$ ) as an analytic function in the open right half-plane and to extend it to a neighborhood of $\lambda=0$. In Section 3, we express $\mathrm{G}_{\lambda}$ as a sum of suitably chosen solutions of (19), so that the compatibility system at $j=l$ allows us to establish that $\mathrm{G}_{\lambda}$ is meromorphic (if the shock is compressive) with $\lambda=0$ as its only pole in a carefully chosen neighborhood of 0 and thus to find bounds of $G_{\lambda}$ for small and medium values of $\lambda$. We do not have to consider large values of $\lambda$ since, the scheme having a finite propagation speed, $\mathrm{G}(n, l, j)$ vanishes for $n+1 \leq|l-j|$.

In Section 4, we use the inverse Laplace transform to get bounds of $\mathrm{G}$ :

$$
\mathrm{G}(n, l, j)=\frac{1}{2 i \pi} \int_{\Gamma} \mathrm{e}^{\lambda n} \mathrm{G}_{\lambda}(l, j) \mathrm{d} \lambda,
$$

where $\Gamma$ is a path of $\mathcal{S}$ that lies a priori in the open right half-plane. But thanks to the Cauchy formula, this path can be changed to better suit the behavior of $\mathrm{G}_{\lambda}$ : we choose moving contours, that depend on $n, l$, and $j$, and that may lie partly in the left half-plane, as in the work of Zumbrun and Howard [27]. We take a particular care to deal with the compressive shocks, since in these cases the Laplace transform of G has a pole (of finite order) at the origin: the choice of contours may then yield a stationary residual term, which is a projection on the eigenspace associated with the eigenvalue 1 of $L^{\mathrm{s}}$, as stated in Theorem 1.1.

A similar technique can be applied to the Lax-Wendroff scheme, but the computations are rather more complicated.

Let us now consider the boundary layer setting: let a $d \times d$ system of conservation laws on the half-line $\mathbb{R}^{+}$ with initial datum be

$$
\begin{aligned}
u_{t}+f(u)_{x} & =0, x \geq 0 \\
u(x, 0) & =\mathrm{u}_{0}(x), x \geq 0 .
\end{aligned}
$$


We are still considering the MLF scheme, but now we consider a mesh of $\mathbb{R}^{+}$that consists of cells $\mathcal{M}_{j}=$ $(j h,(j+1) h]$ of size $h$ with $j \in \mathbb{N}$. We denote by $k$ the time step and $t^{n}=n k, n \in \mathbb{N}$. The nonlinear evolution operator associated with the MLF scheme is still denoted by $\mathcal{N}$ (see Eqs. (4) and (5)).

We assume as usual that $\mathrm{d} f(u)$ is symmetrizable, that is,

K 1. There exist smooth matrices $P$, being invertible, and $D$, being diagonal, such that $\mathrm{d} f(u)=P(u) D(u) P(u)^{-1}$.

Boundary conditions at $x=0$ are needed: choosing Neuman's condition leads to small boundary layers of size $h$ and Dirichlet's condition to large boundary layers of size 1. Here we consider Dirichlet's boundary conditions: the numerical problem becomes

$$
\begin{aligned}
u^{n+1} & =\mathcal{N}\left(u^{n}\right), u=\left(u_{j}\right)_{j \in \mathbb{N}}, \\
u_{j}^{0} & =\frac{1}{\left|\mathcal{M}_{j}\right|} \int_{\mathcal{M}_{j}} \mathrm{u}_{0}(x) \mathrm{d} x, j \geq 1, \\
u_{0}^{n} & =0, n \in \mathbb{N},
\end{aligned}
$$

where $\mathcal{L}$ is once again the MLF (nonlinear) operator.

We also assume that the boundary is non-characteristic, that is,

K 2. The value 0 is never an eigenvalue of $\mathrm{d} f(u)$,

and that the CFL condition is satisfied along the boundary layer profile

K 3. $\sup _{u \in \mathcal{U}} \mathrm{r}(\mathrm{d} f(u))<2 D<\frac{k}{h}$, $\mathrm{r}$ denoting the spectral radius.

Under a smallness assumption, Chainais-Hillairet and Grenier [4] proved the convergence of the numerical solutions to the solutions of $(21,22)$ with boundary conditions

$$
u(0, t) \in \mathcal{C}_{\text {num }}
$$

where $\mathcal{C}_{\text {num }}$ is the set of vectors $w$ such that there exists a solution $v=\left(v_{j}\right)_{j \in \mathbb{N}}$ to

$$
\mathcal{F}\left(w+v_{j}, u+v_{j+1}\right)=f(w), v_{0}=w, v_{+\infty}=0,
$$

$\mathcal{F}$ being the numerical flux of $\mathcal{N}$. This result is similar to the one obtained for the Godunov scheme by Gisclon and Serre [8]. This condition is analogous to setting on the solution to be zero on the entering characteristics when the flux is linear.

This situation is the discrete analogue of the non-viscous limit of

$$
\begin{aligned}
u_{t}^{\varepsilon}+f\left(u^{\varepsilon}\right)_{x}-\varepsilon u_{x x}^{\varepsilon} & =0, \\
u^{\varepsilon}(0) & =0,
\end{aligned}
$$

as $\varepsilon$ tends to 0 , the numerical viscosity of the MLF scheme being $D h$. Indeed, as proved in [7], under specific assumptions, the solutions $u^{\varepsilon}$ tend to solutions of

$$
\begin{aligned}
u_{t}+f(u)_{x} & =0, \\
u(0, t) & \in \mathcal{C}_{\mathrm{vis}},
\end{aligned}
$$

where $\mathcal{C}_{\text {vis }}$ is the subset of $\mathbb{R}^{d}$ consisting of vectors $w$ that satisfy

$$
\partial_{x} f(v+w)=\partial_{x x} v \text { for } x>0, v \underset{x \rightarrow \pm \infty}{\longrightarrow} 0, v(0)=-w
$$

The stability of these solutions has been proved under a smallness assumption by Grenier and Guès [11] and under some spectral assumptions by Grenier and Rousset [12]. 
We assume here the existence of a boundary layer profile, that is,

K 4. There exists a sequence $\bar{u}^{\mathrm{bl}}=\left(\bar{u}_{j}^{\mathrm{bl}}\right)_{j \in \mathbb{N}}$ that satisfies

$$
\begin{gathered}
\mathcal{N}\left(\bar{u}^{\mathrm{bl}}\right)=\bar{u}^{\mathrm{bl}}, \\
\bar{u}^{\mathrm{bl}} \underset{j \rightarrow+\infty}{\longrightarrow} u^{+} \\
\bar{u}_{0}=0
\end{gathered}
$$

where $u^{+}$belongs to $\mathcal{C}_{\text {num. }}$.

Similarly as in the shock profile setting, we assume that:

K 5. The eigenvalues of $\mathrm{d} f\left(u^{+}\right)$are distinct.

Next, we linearize the operator $\mathcal{N}$ about $\bar{u}_{\text {bl }}$ and, denoting by $L^{\text {bl }}$ the linearized operator, we study

$$
\begin{aligned}
& u^{n+1}-L^{\mathrm{bl}} u^{n}=\tilde{u}^{n}, \forall n \geq 0, \\
& u_{j}^{n} \underset{j \rightarrow+\infty}{\longrightarrow} 0, \forall n \geq 0, \\
& u^{0}=\mathbf{u}, \\
& u_{0}^{n}=0, \forall n \geq 0,
\end{aligned}
$$

where $\tilde{u}$ and $\mathbf{u}$ are given sequences. The Green's function of $L^{\mathrm{bl}}$ is defined similarly as the one of $L^{\mathrm{s}}$ and satisfies the following problem, $l$ being given in $\mathbb{N}$,

$$
\begin{aligned}
& \mathrm{G}(n+1, l, j)-L^{\mathrm{bl}} \mathrm{G}(n, l, j)=0, \\
& \mathrm{G}(n, l, j) \underset{j \rightarrow+\infty}{\longrightarrow} 0, \\
& \mathrm{G}(0, l, j)=\delta_{l j} I_{d}, \\
& \mathrm{G}(n, l, 0)=0 .
\end{aligned}
$$

Similarly as in the case of shock profiles, the formal solution of (27)-(30) is given by

$$
u_{j}^{n}=\sum_{l=0}^{+\infty} \mathrm{G}(n, l, j) \mathbf{u}_{l}+\sum_{\bar{n}=0}^{n-1} \sum_{l=0}^{+\infty} \mathrm{G}(n-\bar{n}, l, j) \tilde{u}_{l}^{\bar{n}}, \text { for } j \in \mathbb{Z}, n \geq 1 .
$$

Our aim is to obtain analogous estimates as the ones Grenier and Rousset found in the continuous viscous case [12]. The construction of the Evans function $\mathcal{D}$ in the case of numerical boundary layers is much like what we described for the shock profiles, the main difference being that we need $\left(v_{1}(\mu, j), \ldots, v_{d}(\mu, j)\right)($ resp. $\left.\left(v_{d+1}(\mu, j), \ldots, v_{2 d}(\mu, j)\right)\right)$ a basis of the space of solutions of

$$
\left(L^{\mathrm{bl}}-\mu\right) v=0
$$

satisfying $v_{q}(\mu, 0)=0$ for $q \in\{1, \ldots, d\}$ (resp. $v_{q}(\mu, j)$ tends to 0 as $j$ tends to $+\infty$ ) instead of the condition at $j=-\infty$ that we used in the shock profile setting. For $|\mu|>1$, we define the Evans function as

$$
\mathcal{D}(\mu, j)=\operatorname{det}\left(\begin{array}{cccccc}
v_{1}(\mu, j) & \ldots & v_{d}(\mu, j) & v_{d+1}(\mu, j) & \ldots & v_{2 d}(\mu, j) \\
\Delta v_{1}(\mu, j) & \ldots & \Delta v_{d}(\mu, j) & \Delta v_{d+1}(\mu, j) & \ldots & \Delta v_{2 d}(\mu, j)
\end{array}\right) .
$$

As in the case of shock profiles, the zeroes of $\mathcal{D}(\cdot, j)$ correspond to unstable eigenvalues of $L^{\mathrm{bl}}$. Since the eigenvalues of modulus larger than 1 induce instability, we assume that there are none in $|\mu|>1$ and extending 
$\mathcal{D}$ to a neighborhood of the circle $|\mu|=1$, we also assume that 1 is not an eigenvalue of $L^{\text {bl }}$, that is,

K 6. The Evans function does not vanish outside the open unit-disc, that is

$$
\mathcal{D}(\mu, j) \neq 0, \forall j \in \mathbb{N}, \forall \mu,|\mu| \geq 1
$$

Performing the same kind of analysis as in the case of shock profiles, we obtain the following theorem:

Theorem 1.2 (Green's function of a pure boundary layer). Assuming (K1)-(K5), the Green's function of (27)(30) behaves as follows:

$$
\begin{aligned}
& \text { given } \vec{e} \in \mathbb{R}^{d} \\
& \mathrm{G}(n, l, j) \cdot \vec{e}=\sum_{q / a_{q}>0} \frac{1}{\sqrt{n}} O\left(\exp \left(-\frac{\left(j-a_{q} n\right)^{2}}{M n}\right)\right) r_{q}+O\left(\mathrm{e}^{-\gamma n} \exp \left(-\frac{(l-j)^{2}}{M n}\right)\right), \forall l, j \in \mathbb{N}, \\
& \Delta_{j} \mathrm{G}(n, l, j) \cdot \vec{e}=\sum_{q / a_{q}>0} \frac{1}{n} O\left(\exp \left(-\frac{\left(j-a_{q} n\right)^{2}}{M n}\right)\right) r_{q}+O\left(\mathrm{e}^{-\gamma n} \exp \left(-\frac{(l-j)^{2}}{M n}\right)\right), \forall l, j \in \mathbb{N},
\end{aligned}
$$

where

- we denote by $a_{1}<\ldots<a_{d}$ the eigenvalues of $(k / h) \mathrm{d} f\left(u^{+}\right)$and by $\left(r_{q}\right)_{q \in\{1, \ldots, d\}}$ some associated eigenvectors;

- the notation $q / a_{q}>0$ addresses the indices $q \in\{1, \ldots, d\}$ such that $a_{q}$ is positive;

- all the constants are locally bounded with respect to $l$ and uniformly bounded with respect to $n, j$ and $M, \gamma$ are positive.

This result is analogous to the one obtained in the viscous case developed in Section 4 of [12]. Physically, Theorem 1.2 states that the Dirac mass splits upon both the outgoing and entering characteristics. Along each outgoing characteristic, a Gaussian-shaped wave enters the domain with an asymptotic speed that is equal to the eigenvalue of $\mathrm{d} f\left(u^{+}\right)$corresponding to the considered characteristic multiplied by the ratio $k / h$. These waves are damped by the numerical viscosity. Each time a wave that is carried by an entering characteristic reaches the boundary, new waves behaving as described above leave the boundary to enter the domain.

We do not give the proof of Theorem 1.2 hereafter, since it is very close to the one of the shock wave setting. Note however that the main differences appear in the statement of the problems and that once they have been linearized about respectively a shock profile and a boundary layer, they are remarkably similar.

Finding bounds on the Green's function is a valuable step in the search for nonlinear stability as it was proved by Grenier and Rousset in [12] for the viscous boundary layers. As a matter of fact, the very nice work of Mascia and Zumbrun [21] on the stability of relaxation shocks should allow the use of Theorem 1.2 to get a result of nonlinear stability.

\section{Construction of an Evans function}

From now on, we only consider the case of the shock profiles and consequently, we rename the linearized operator $L^{\mathrm{s}}$ in $L$. We aim here to construct a basis of the spaces consisting of solutions $v$ of the equation

$$
\left(L-\mathrm{e}^{\lambda}\right) v=0
$$

that decrease to 0 as $j$ tends to $\pm \infty$. 
Noting $\Delta v_{j-1}:=v_{j}-v_{j-1}$ for any sequence $\left(v_{j}\right)_{j \in \mathbb{Z}}$ and rewriting (17) with no right-hand term as a first-order recurrence on $V_{j}:=\left(v_{j}, \Delta v_{j}\right)^{T}$, we get

$$
\begin{aligned}
V_{j} & =\left(\left(\frac{F_{j+1}}{2}-D I_{d}\right)^{-1}\left(1-\mathrm{e}^{\lambda}+\frac{F_{j-1}-F_{j+1}}{2}\right)\left(\frac{F_{j+1}}{2}-D I_{d}\right)^{-1}\left(\left(1-\mathrm{e}^{\lambda}-D\right) I_{d}-\frac{F_{j+1}}{2}\right)\right) V_{j-1} \\
& =: \mathbb{A}_{j}(\lambda) V_{j-1}, j \in \mathbb{Z} .
\end{aligned}
$$

\subsection{Asymptotic behavior of the solutions of the homogeneous recurrence}

Let us now study the asymptotic behaviors of the solutions of (35). As $j$ tends to $\pm \infty$, the sequence of matrices $\left(\mathbb{A}_{j}(\lambda)\right)$ tends to

$$
\mathbb{A}^{ \pm}(\lambda):=\left(\begin{array}{c}
I_{d} \\
\left(\mathrm{e}^{\lambda}-1\right)\left(D I_{d}-\frac{F^{ \pm}}{2}\right)^{-1}\left(D I_{d}-\frac{F^{ \pm}}{2}\right)^{-1}\left(\mathrm{e}^{\lambda}-1+D+\frac{F^{ \pm}}{2}\right)
\end{array}\right) .
$$

Using the strict hyperbolicity of (1) at the end states $u^{ \pm}$, we obtain the following formula of the characteristic polynomial of $\mathbb{A}^{ \pm}(\lambda)$

$$
\Pi^{ \pm}(\mathrm{w}, \lambda):=\prod_{q=1}^{d}\left(\mathrm{w}^{2}+2\left(2 D-a_{q}^{ \pm}\right)^{-1}\left(1-2 D-\mathrm{e}^{\lambda}\right) \mathrm{w}+\left(2 D-a_{q}^{ \pm}\right)^{-1}\left(2 D+a_{q}^{ \pm}\right)\right) .
$$

Thus, the eigenvalues of $\mathbb{A}^{ \pm}(\lambda)$ are

$$
\mathrm{w}_{\varepsilon, q}^{ \pm}(\lambda)=\frac{1}{\left(2 D-a_{q}^{ \pm}\right)}\left(2 D+\mathrm{e}^{\lambda}-1+\varepsilon \sqrt{\left(\mathrm{e}^{\lambda}-1\right)^{2}+4 D\left(\mathrm{e}^{\lambda}-1\right)+\left(a_{q}^{ \pm}\right)^{2}}\right), \varepsilon= \pm 1,
$$

for $q \in\{1, \ldots, d\}$ and

$$
R_{q, \varepsilon}^{ \pm}(\lambda)=\left(\begin{array}{c}
r_{q}^{ \pm} \\
\left(\mathrm{w}_{\varepsilon, q}^{ \pm}(\lambda)-1\right) r_{q}^{ \pm}
\end{array}\right)
$$

are associated eigenvectors. The eigenelements $\mathrm{w}_{\varepsilon, q}^{ \pm}$and $R_{\varepsilon, q}^{ \pm}$associated with $a_{q}^{ \pm}$are holomorphic if $\lambda \in \mathbb{C} \backslash$ $(-\infty, \Lambda]$, with

$$
\Lambda:=\min _{q \in\{1, \ldots, d\}} \ln \left(1-2 D\left(1-\sqrt{1-\frac{\left(a_{q}^{ \pm}\right)^{2}}{4 D^{2}}}\right)\right) .
$$

We denote by

$$
\mathcal{E}_{q}^{ \pm}:=\left\{\lambda \in \mathbb{C} / \exists \theta \in \mathbb{R} / \mathrm{e}^{\lambda}=1-2 D(1-\cos (\theta))-i a_{q}^{ \pm} \sin (\theta)\right\}
$$

the set of complex points $\lambda$ such that the modulus of a solution $w$ of (36) is 1 . Note that, since $2 D<1$ and $\left|a_{q}^{ \pm}\right|<1,(-\infty, \Lambda]$ and $\mathcal{E}_{q}^{ \pm}$lie in the left half-plane. Besides, $\mathcal{E}_{q}^{ \pm}$only intersects the imaginary axis at $\lambda=0$. From now on, $\mathcal{E}$ will denote the biggest of the $\mathcal{E}_{a}$, i.e. $\mathcal{E}_{\max \left(a_{q}^{ \pm}\right)}$. Let $\Omega$ be the (open) connected component of $\mathcal{S} \backslash(\mathcal{E} \cup(-\infty, \Lambda])$ containing $+\infty$ and consider $\lambda \in \Omega$ (see Fig. 2). 


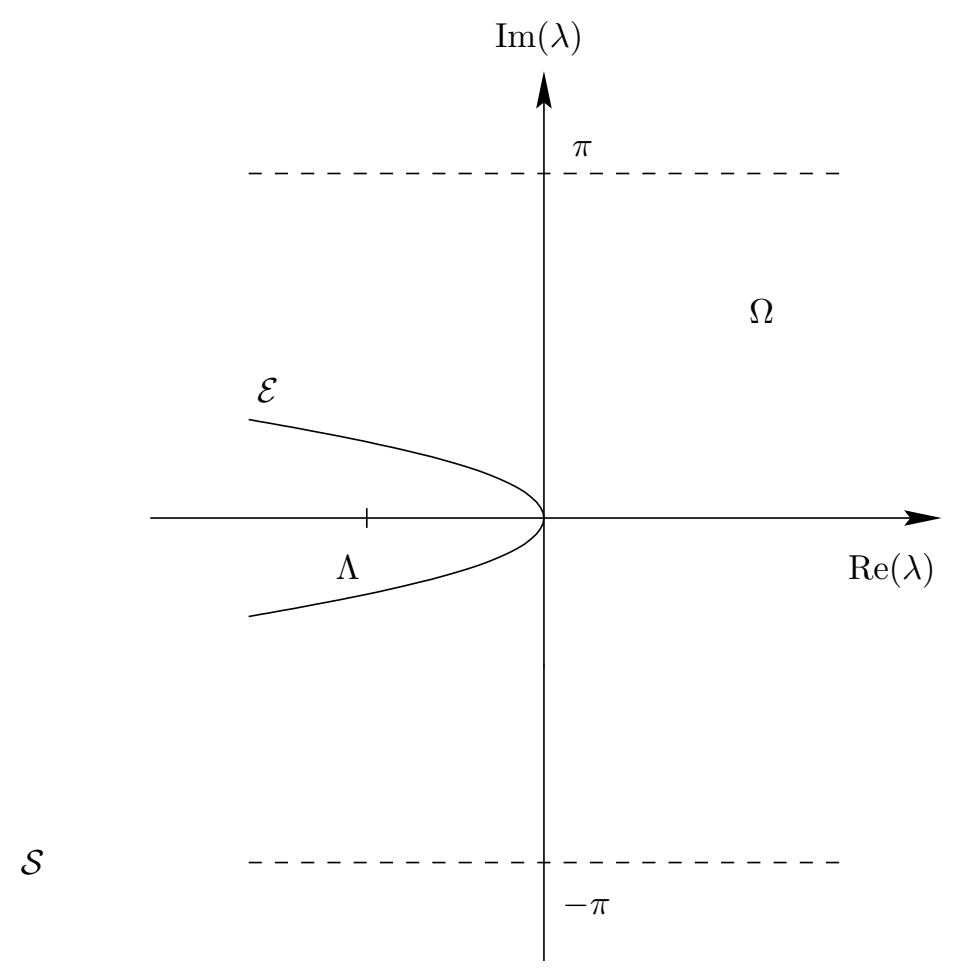

Figure 2. Spectrum of $L$ in the complex plane.

Lemma 2.1. Let $\lambda \in \Omega$. The matrices $\mathbb{A}^{ \pm}(\lambda)$ have $d$ eigenvalues of modulus strictly larger than 1 and $d$ eigenvalues of modulus strictly less than 1 .

Proof. We drop temporarily the superscript \pm for the sake of simplicity: $a_{q}$ denotes any eigenvalue of $F^{ \pm}$.

Developing $\mathrm{w}_{\varepsilon, q}$ in a neighborhood of $\lambda=0$, we obtain

$$
\mathrm{w}_{-\operatorname{sgn}\left(a_{q}\right), q}=1-\frac{\lambda}{a_{q}}-\frac{1}{2 a_{q}}\left(1-\frac{2 D+a_{q}}{a_{q}^{2}}\right) \lambda^{2}+O\left(|\lambda|^{3}\right)
$$

and

$$
\mathrm{w}_{\operatorname{sgn}\left(a_{q}\right), q}=\frac{2 D+a_{q}}{2 D-a_{q}}+O(|\lambda|) .
$$

We see at once that, for small real $\lambda$, we have

$$
\left|\mathrm{w}_{\operatorname{sgn}\left(a_{q}\right), q}\right|>1 \Leftrightarrow\left(\lambda>0 \text { and } a_{q}<0\right)
$$

Consequently, since $q \in\{1, \ldots, d\}$, there are $d$ eigenvalues of modulus larger than 1 and $d$ eigenvalues of modulus less than 1 for small positive $\lambda$. Since the definitions of $\mathcal{E}$ and $\Omega$ imply that there are no eigenvalue of modulus 1 if $\lambda \in \Omega$, the holomorphy of the set of solutions of the characteristic polynomial of $\mathbb{A}(\lambda)$ outside $(-\infty, \Lambda]$ allows us to conclude that $\mathbb{A}(\lambda)$ has $d$ eigenvalues of modulus larger than 1 and $d$ eigenvalues of modulus less than 1 . 
The matrices $\mathbb{A}^{+}(\lambda)$ and $\mathbb{A}^{-}(\lambda)$ have no eigenvalue of modulus 1 in $\Omega$ because of the definition of $\mathcal{E}$. By Theorem 6.1 in the appendix of $[9], \mathbb{A}^{+}(\lambda)$ (resp. $\mathbb{A}^{-}(\lambda)$ ) has an exponential dichotomy on $\mathbb{N}$ (resp. $-\mathbb{N}$ ). Since $\left(\mathbb{A}_{j}(\lambda)\right)_{j \in \mathbb{Z}}$ tends to $\mathbb{A}^{ \pm}(\lambda)$ as $j$ tends to $\pm \infty$ exponentially, we can apply Theorem 6.2 ([9], Appendix) and $\left(\mathbb{A}_{j}(\lambda)\right)_{j}$ has an exponential dichotomy on $\mathbb{N}($ resp. $-\mathbb{N}$ ) of projection $P(\lambda)$ (resp. $Q(\lambda)$ ) and $\operatorname{ker}(P(\lambda))$ (resp. $\operatorname{ker}(Q(\lambda)))$ and $R(P(\lambda))$ (resp. $R(Q(\lambda)))$ are $d$-dimensional (Lem. 2.1). Let

$$
\begin{gathered}
E(\lambda):=\left\{\left(V_{j}\right)_{j \in \mathbb{Z}} / V_{j+1}=\mathbb{A}_{j}(\lambda) V_{j} \text { and } V_{j} \underset{j \rightarrow+\infty}{\longrightarrow} 0\right\}, \\
E_{0}(\lambda):=\left\{V_{0} /\left(V_{j}\right)_{j \in \mathbb{Z}} \in E(\lambda)\right\}, \\
F(\lambda):=\left\{\left(V_{j}\right)_{j \in \mathbb{Z}} / V_{j+1}=\mathbb{A}_{j}(\lambda) V_{j} \text { and } V_{j} \underset{j \rightarrow-\infty}{\longrightarrow} 0\right\}
\end{gathered}
$$

and

$$
F_{0}(\lambda):=\left\{V_{0} /\left(V_{j}\right)_{j \in \mathbb{Z}} \in F(\lambda)\right\} .
$$

If $E_{0}(\lambda) \cap F_{0}(\lambda) \neq\{0\}, \mathrm{e}^{\lambda}$ is an eigenvalue of $L$. Otherwise, we have

$$
R(P(\lambda)) \oplus \operatorname{ker}(Q(\lambda))=\mathbb{C}^{2 d} .
$$

Consequently, using Theorem 6.3 ([9], Appendix), we conclude that the dynamical system (35) has an exponential dichotomy on $\mathbb{Z}$ if and only if $\mathrm{e}^{\lambda}$ is not an eigenvalue of $L$, for all $\lambda \in \Omega$ (see Fig. 2). Furthermore, if we consider a basis $\mathcal{B}_{E(\lambda)}$ (resp. $\mathcal{B}_{F(\lambda)}$ ) of $E(\lambda)$ (resp. $F(\lambda)$ ) consisting of $d$ eigenfunctions of (35) geometrically decreasing towards 0 at $+\infty$ (resp. $-\infty$ ), we conclude at once that the set $\mathcal{B}_{E(\lambda)} \cup \mathcal{B}_{F(\lambda)}$ is a basis of the whole space of solutions of (35) if and only if $\mathrm{e}^{\lambda}$ is not an eigenvalue of $L$. Thus we can define an Evans function $(\lambda, j) \mapsto \mathcal{D}(\lambda, j)$ as a determinant of the elements of $\mathcal{B}_{E(\lambda)} \cup \mathcal{B}_{F(\lambda)}$ taken at the point $j \in \mathbb{Z}$. A most interesting feature of $\mathcal{D}(\cdot, j)$ is its vanishing at the points $\lambda$ such that $\mathrm{e}^{\lambda}$ is an eigenvalue of $L$. Moreover, the function $\mathcal{D}$ is analytic on $\Omega$.

\subsection{Extension to a neighborhood of $\lambda=0$}

Besides, for $\lambda \in D(0, \sigma)$ with $\sigma$ sufficiently small, the asymptotic behaviors of the eigenelements of $\mathbb{A}^{ \pm}(\lambda)$ are given in Tables 1 and 2 where

$$
\zeta_{q}^{ \pm}:=\frac{2 D+a_{q}^{ \pm}}{2 D-a_{q}^{ \pm}}
$$

Thus, in a neighborhood of $\lambda=0$, the matrices $\mathbb{A}^{ \pm}(\lambda)$ are diagonalizable, because the strict hyperbolicity assumption (H2) implies that the $\mathrm{w}_{\varepsilon, q}^{ \pm}$are distinct. Consequently, we can easily choose $\left(\Phi_{q}(\lambda, j)\right)_{q \in\{1, \ldots, d\}}$ (resp. $\left.\left(\Phi_{q}(\lambda, j)\right)_{q \in\{d+1, \ldots, 2 d\}}\right)$ a basis of solutions of (35) which decay towards 0 as $j$ tends to $+\infty$ (resp. $\left.-\infty\right)$, which depend analytically on $\lambda$ for $\lambda \in D(0, \sigma)$ and that can be extended analytically to a neighborhood of $\lambda=0$ such that their behaviors are as follows

$$
\begin{aligned}
& \Phi_{q}(\lambda, j) \underset{j \rightarrow+\infty, \lambda \rightarrow 0}{=}\left(\mathrm{w}_{q}^{+}(\lambda)\right)^{j}\left(R_{q}^{+}(\lambda)+O\left(\omega^{-j}\right)\right), \text { for } q \in\{1, \ldots, d\}, \\
& \Phi_{q}(\lambda, j) \underset{j \rightarrow-\infty, \lambda \rightarrow 0}{=}\left(\mathrm{w}_{q}^{-}(\lambda)\right)^{j}\left(R_{q}^{-}(\lambda)+O\left(\omega^{j}\right)\right), \text { for } q \in\{d+1, \ldots, 2 d\},
\end{aligned}
$$


TABLE 1. Asymptotic behavior as $j \rightarrow-\infty$ and $\lambda \rightarrow 0$.

\begin{tabular}{|c|c|}
\hline \multicolumn{2}{|c|}{$a_{1}^{-}<\ldots<a_{p^{-}-1}^{-}<0$ and $0<a_{p^{-}}^{-}<\ldots<a_{d}^{-}$} \\
\hline $\begin{array}{c}\mathrm{w}_{1}^{-}(\lambda)=\zeta_{1}^{-}+O(|\lambda|) \\
R_{1}^{-}(\lambda)=\left(\begin{array}{c}r_{1}^{-} \\
\left(\zeta_{1}^{-}-1\right) r_{1}^{-}\end{array}\right)+O\left(\begin{array}{l}|\lambda| \\
|\lambda|\end{array}\right)\end{array}$ & $\begin{array}{c}\mathrm{w}_{d+1}^{-}(\lambda)=1-\frac{\lambda}{a_{1}^{-}}+O\left(|\lambda|^{2}\right) \\
R_{d+1}^{-}(\lambda)=\left(\begin{array}{c}r_{1}^{-} \\
-\frac{\lambda}{a_{1}^{-}} r_{1}^{-}\end{array}\right)+O\left(\begin{array}{c}|\lambda| \\
|\lambda|^{2}\end{array}\right)\end{array}$ \\
\hline & $\vdots$ \\
\hline $\begin{array}{c}\mathrm{w}_{p^{-}-1}^{-}(\lambda)=\zeta_{p^{-}-1}^{-}+O(|\lambda|) \\
R_{p^{-}-1}^{-}(\lambda)=\left(\begin{array}{c}r_{1}^{-} \\
\left(\zeta_{p^{-}-1}^{-}-1\right) r_{p^{-}-1}^{-}\end{array}\right)+O\left(\begin{array}{l}|\lambda| \\
|\lambda|\end{array}\right)\end{array}$ & $\begin{array}{c}\mathrm{w}_{d+p^{-}-1}^{-}(\lambda)=1-\frac{\lambda}{a_{p^{-}-1}^{-}} r_{p^{-}-1}^{-}+O\left(|\lambda|^{2}\right) \\
R_{d+p^{-}-1}^{-}(\lambda)=\left(\begin{array}{c}r_{p^{-}-1}^{-} \\
-\frac{\lambda}{a_{p^{-}-1}^{-}} r_{p^{-}-1}^{-}\end{array}\right)+O\left(\begin{array}{c}|\lambda| \\
|\lambda|^{2}\end{array}\right)\end{array}$ \\
\hline $\begin{array}{c}\mathrm{w}_{p^{-}}^{-}(\lambda)=1-\frac{\lambda}{a_{p^{-}}^{-}}+O\left(|\lambda|^{2}\right) \\
R_{p^{-}}^{-}(\lambda)=\left(\begin{array}{c}r_{p^{-}}^{-} \\
-\frac{\lambda}{a_{p^{-}}^{-}} r_{p^{-}}^{-}\end{array}\right)+O\left(\begin{array}{c}|\lambda| \\
|\lambda|^{2}\end{array}\right)\end{array}$ & $\begin{array}{c}\mathrm{w}_{d+p^{-}}^{-}(\lambda)=\zeta_{p^{-}}^{-}+O(|\lambda|) \\
R_{d+p^{-}}^{-}(\lambda)=\left(\begin{array}{c}r_{p^{-}}^{-} \\
\left(\zeta_{p^{-}}^{-}-1\right) r_{p^{-}}^{-}\end{array}\right)+O\left(\begin{array}{l}|\lambda| \\
|\lambda|\end{array}\right)\end{array}$ \\
\hline & $\vdots$ \\
\hline $\begin{array}{c}\mathrm{w}_{d}^{-}(\lambda)=1-\frac{\lambda}{a_{d}^{-}}+O\left(|\lambda|^{2}\right) \\
R_{d}^{-}(\lambda)=\left(\begin{array}{c}r_{d}^{-} \\
-\frac{\lambda}{a_{d}^{-}} r_{d}^{-}\end{array}\right)+O\left(\begin{array}{c}|\lambda| \\
|\lambda|^{2}\end{array}\right)\end{array}$ & $\begin{array}{c}\mathrm{w}_{2 d}^{-}(\lambda)=\zeta_{d}^{-}+O(|\lambda|) \\
R_{2 d}^{-}(\lambda)=\left(\begin{array}{c}r_{d}^{-} \\
\left(\zeta_{d}^{-}-1\right) r_{d}^{-}\end{array}\right)+O\left(\begin{array}{l}|\lambda| \\
|\lambda|\end{array}\right)\end{array}$ \\
\hline
\end{tabular}

with $\omega>1$. Consequently, the Evans function $\mathcal{D}$ can also be analytically extended with respect to $\lambda$ to a neighborhood of $\lambda=0$ because of (41), so that it can be expressed explicitly in $D(0, \sigma)$ as the following determinant

$$
\mathcal{D}(\lambda, l)=\operatorname{det}\left(\Phi_{1}(\lambda, l), \ldots, \Phi_{d}(\lambda, l), \Phi_{d+1}(\lambda, l), \ldots, \Phi_{2 d}(\lambda, l)\right) .
$$

Rewriting the linearized scheme (8) in the conservative form, we have

$$
\left(\mathrm{e}^{\lambda}-1\right) v_{j}+\left(\frac{F_{j}}{2}+D I_{d}\right) v_{j}+\left(\frac{F_{j+1}}{2}-D I_{d}\right) v_{j+1}=\left(\frac{F_{j-1}}{2}+D I_{d}\right) v_{j-1}+\left(\frac{F_{j}}{2}-D I_{d}\right) v_{j}
$$

Thus, at $\lambda=0$, if $\left(v_{j}\right)_{j \in \mathbb{Z}}$ decreases towards 0 as $j$ tends to $\pm \infty$, taking the limit of the right member of (42) at $j= \pm \infty$, we get

$$
v_{j+1}-v_{j}=\left(\left(2 D I_{d}-F_{j+1}\right)^{-1}\left(2 D I_{d}+F_{j}\right)-I_{d}\right) v_{j}=: M_{j} v_{j}
$$

Denoting generically $\Phi=\left(\Phi^{\mathrm{I}}, \Phi^{\mathrm{II}}\right)^{T}$, with $\Phi^{\mathrm{I}}, \Phi^{\mathrm{II}} \in \mathbb{C}^{d}$, we then apply $(43)$ to $\Phi_{q}^{\mathrm{I}}(0, \cdot)$ for $q \in\left\{1, \ldots, p^{+}\right\} \cup\{d+$ $\left.p^{-}, \ldots, 2 d\right\}$, according to Tables 1 and 2 and to (41). Besides, if $\lambda=0, \mathrm{w}=1$ is an eigenvalue of multiplicity $d$ 
TABLE 2. Asymptotic behavior as $j \rightarrow+\infty$ and $\lambda \rightarrow 0$.

\begin{tabular}{|c|c|}
\hline \multicolumn{2}{|c|}{$a_{1}^{+}<\ldots<a_{p^{+}}^{+}<0$ and $0<a_{p^{+}+1}^{+}<\ldots<a_{d}^{+}$} \\
\hline $\begin{array}{c}\mathrm{w}_{1}^{+}(\lambda)=\zeta_{1}^{+}+O(|\lambda|) \\
R_{1}^{+}(\lambda)=\left(\begin{array}{c}r_{1}^{+} \\
\left(\zeta_{1}^{+}-1\right) r_{1}^{+}\end{array}\right)+O\left(\begin{array}{l}|\lambda| \\
|\lambda|\end{array}\right)\end{array}$ & $\begin{array}{c}\mathrm{w}_{d+1}^{+}(\lambda)=1-\frac{\lambda}{a_{1}^{+}}+O\left(|\lambda|^{2}\right) \\
R_{d+1}^{+}(\lambda)=\left(\begin{array}{c}r_{1}^{+} \\
-\frac{\lambda}{a_{1}^{+}} r_{1}^{+}\end{array}\right)+O\left(\begin{array}{c}|\lambda| \\
|\lambda|^{2}\end{array}\right)\end{array}$ \\
\hline $\begin{array}{c}\mathrm{w}_{p^{+}}^{+}(\lambda)=\zeta_{p^{+}}^{+}+O(|\lambda|) \\
R_{p^{+}}^{+}(\lambda)=\left(\begin{array}{c}r_{1}^{+} \\
\left(\zeta_{p^{+}}^{+}-1\right) r_{p^{+}}^{+}\end{array}\right)+O\left(\begin{array}{c}|\lambda| \\
|\lambda|\end{array}\right)\end{array}$ & $\begin{array}{c}\mathrm{w}_{d+p^{+}}^{+}(\lambda)=1-\frac{\lambda}{a_{p^{+}}^{+}} r_{p^{+}}^{+}+O\left(|\lambda|^{2}\right) \\
R_{d+p^{+}}^{+}(\lambda)=\left(\begin{array}{c}r_{p^{+}}^{+} \\
-\frac{\lambda}{a_{p^{+}}^{+}} r_{p^{+}}^{+}\end{array}\right)+O\left(\begin{array}{c}|\lambda| \\
|\lambda|^{2}\end{array}\right)\end{array}$ \\
\hline $\begin{array}{c}\mathrm{w}_{p^{+}+1}^{+}(\lambda)=1-\frac{\lambda}{a_{p^{+}+1}^{+}}+O\left(|\lambda|^{2}\right) \\
R_{p^{+}+1}^{+}(\lambda)=\left(\begin{array}{c}r_{p^{+}+1}^{+} \\
-\frac{\lambda}{a_{p^{+}+1}^{+}} r_{p^{+}+1}^{+}\end{array}\right)+O\left(\begin{array}{c}|\lambda| \\
|\lambda|^{2}\end{array}\right)\end{array}$ & $\begin{array}{c}\mathrm{w}_{d+p^{+}+1}^{+}(\lambda)=\zeta_{p^{+}+1}^{+}+O(|\lambda|) \\
R_{d+p^{+}+1}^{+}(\lambda)=\left(\begin{array}{c}r_{p^{+}+1}^{+} \\
\left(\zeta_{p^{+}+1}^{+}-1\right) r_{p^{+}+1}^{+}\end{array}\right)+O\left(\begin{array}{l}|\lambda| \\
|\lambda|\end{array}\right)\end{array}$ \\
\hline $\begin{array}{c}\mathrm{w}_{d}^{+}(\lambda)=1-\frac{\lambda}{a_{d}^{+}}+O\left(|\lambda|^{2}\right) \\
R_{d}^{+}(\lambda)=\left(\begin{array}{c}r_{d}^{+} \\
-\frac{\lambda}{a_{d}^{+}} r_{d}^{+}\end{array}\right)+O\left(\begin{array}{c}|\lambda| \\
|\lambda|^{2}\end{array}\right)\end{array}$ & $\begin{array}{c}\mathrm{w}_{2 d}^{+}(\lambda)=\zeta_{d}^{+}+O(|\lambda|) \\
R_{2 d}^{+}(\lambda)=\left(\begin{array}{c}r_{d}^{+} \\
\left(\zeta_{d}^{+}-1\right) r_{d}^{+}\end{array}\right)+O\left(\begin{array}{l}|\lambda| \\
|\lambda|\end{array}\right)\end{array}$ \\
\hline
\end{tabular}

of $\mathbb{A}^{ \pm}(\lambda)$, that is $\Phi_{p+1}(0, \cdot), \ldots, \Phi_{d+p-1}(0, \cdot)$ are constant and we can set

$$
\begin{aligned}
& \Phi_{p^{+}+1}(0, j)=\left(\begin{array}{c}
r_{p^{+}+1}^{+} \\
0
\end{array}\right), \ldots, \Phi_{d}(0, j)=\left(\begin{array}{c}
r_{d}^{+} \\
0
\end{array}\right), \\
& \Phi_{d+1}(0, j)=\left(\begin{array}{c}
r_{1}^{-} \\
0
\end{array}\right), \ldots, \Phi_{d+p^{-}-1}(0, j)=\left(\begin{array}{c}
r_{p^{-}-1}^{-} \\
0
\end{array}\right) .
\end{aligned}
$$

Thus

$\mathcal{D}(0, j)=\operatorname{det}\left[\begin{array}{cccccccccccc}\Phi_{1}^{\mathrm{I}}(0, j) & \ldots & \Phi_{p^{+}}^{\mathrm{I}}(0, j) & r_{p^{+}+1}^{+} & \ldots & r_{d}^{+} & r_{1}^{-} & \ldots & r_{p^{-}-1}^{-} & \Phi_{d+p^{-}}^{\mathrm{I}}(0, j) & \ldots & \Phi_{2 d}^{\mathrm{I}}(0, j) \\ M_{j} \Phi_{1}^{\mathrm{I}}(0, j) & \ldots & M_{j} \Phi_{p^{+}}^{\mathrm{I}}(0, j) & 0 & \ldots & 0 & 0 & \ldots & 0 & M_{j} \Phi_{d+p^{-}}^{\mathrm{I}}(0, j) & \ldots & M_{j} \Phi_{2 d}^{\mathrm{I}}(0, j)\end{array}\right]$.

We can see at once that the kernel of the matrix inside the determinant (45) is $\varpi+1$-dimensional, which means that 1 is necessarily an eigenvalue of $L$ if the shock $\left(u^{-}, u^{+}\right)$is compressive. Moreover, for $\varpi \geq 0$, without loss of generality, we can set

$$
\Phi_{p^{+-}-m}^{\mathrm{I}}(0, \cdot)=\Phi_{d+p^{-}+m}^{\mathrm{I}}(0, \cdot)=: \Psi_{m}(j), \text { for } m \in\{0, \ldots, \varpi\} .
$$

Remark 2.1. If the discontinuity is undercompressive, $\mathcal{D}(\lambda, \cdot)$ does not necessarily vanish at $\lambda=0$, contrary to the continuous case. Indeed, the discrete derivative of a shock profile of $\mathcal{N}$ does not belong, in general, to the kernel of $L-1$. 
Proposition 2.1. For $m \in\{0, \ldots, \varpi\}$, the $m$ th derivative of the Evans function vanishes at $\lambda=0$ and its $(\varpi+1)$ th derivative is

$$
\frac{\partial^{\varpi+1} \mathcal{D}}{\partial \lambda^{\varpi+1}}(0, j)=(-1)^{p^{+}} \operatorname{det}\left(M_{j}\right) \mathcal{W}(j) \phi_{j}^{\varpi}\left(S_{0}, \ldots, S_{q}\right), \forall j \in \mathbb{Z}
$$

where

$$
\begin{aligned}
\mathcal{W}(j):= & \operatorname{det}\left(\Phi_{1}^{\mathrm{I}}(0, j), \ldots, \Phi_{p^{+}-\varpi-1}^{\mathrm{I}}(0, j), \Psi_{0}(j), \ldots, \Psi_{\varpi}(j), \Phi_{d+p^{-}+\varpi+1}^{\mathrm{I}}(0, j), \ldots, \Phi_{2 d}^{\mathrm{I}}(0, j)\right) \\
\phi_{j}^{\varpi}:= & \mathbb{C}^{\varpi+1} \longrightarrow \mathbb{C} \\
& \left(\vec{e}_{0}, \ldots, \vec{e}_{\varpi}\right) \mapsto \operatorname{det}\left(r_{1}^{-}, \ldots, r_{p^{-}-1}, 2\left(F_{j}+F_{j+1}\right)^{-1} \vec{e}_{0}, \ldots, 2\left(F_{j}+F_{j+1}\right)^{-1} \vec{e}_{\varpi}, r_{p^{+}+1}^{+}, \ldots, r_{d}^{+}\right)
\end{aligned}
$$

and

$$
S_{m}:=\sum_{j \in \mathbb{Z}} \Psi_{m}(j), \forall m \in\{0, \ldots, \varpi\}
$$

Proof. The computation in the case $\varpi=-1$ is straightforward from (45).

Consider now the case $\varpi \geq 0$.

We see at once that, since the rank of the matrix inside the determinant (45) id $d-\varpi-1$, at least two identical columns appear in the $m$ th derivative of the Evans function at $\lambda=0$ for $m \in\{0, \ldots, \varpi+1\}$, so it vanishes.

Using the recurrence principle, a classical computation $[1,6,10,28]$ gives

$$
\begin{aligned}
\frac{\partial^{\varpi+1} \mathcal{D}}{\partial \lambda^{\varpi+1}}(0, j)=\operatorname{det}\left[\Phi_{1}(0, j), \ldots, \Phi_{d+p^{-}-1}(0, j),\right. & \\
\left(\frac{\partial \Phi_{d+p^{-}}}{\partial \lambda}-\frac{\partial \Phi_{p^{+}}}{\partial \lambda}\right)(0, j), \ldots,\left(\frac{\partial \Phi_{d+p^{-}+\varpi}}{\partial \lambda}-\frac{\partial \Phi_{p^{+}-\varpi}}{\partial \lambda}\right)(0, j), & \left.\Phi_{d+p^{-}+\varpi+1}(0, j), \ldots, \Phi_{2 d}(0, j)\right] .
\end{aligned}
$$

Since $\lambda \mapsto \Phi_{p^{+}-m}(\lambda, \cdot)$ and $\lambda \mapsto \Phi_{d+p^{-+} m}(\lambda, \cdot)$ are analytic in $\Omega \cup D(0, \sigma)$ for $m \in\{0, \ldots, \varpi\}, z_{p^{+}-m}(\cdot):=$ $\left(\partial \Phi_{p^{+-}-m}^{\mathrm{I}} / \partial \lambda\right)(0, \cdot)$ and $z_{d+p^{-}+m}(\cdot):=\left(\partial \Phi_{d+p^{-}+m}^{\mathrm{I}} / \partial \lambda\right)(0, \cdot)$ satisfy the same discrete dynamical system, which is obtained by deriving (42) with respect to $\lambda$ and taking $\lambda=0$ :

$$
\Psi_{m}(j)+\left(\frac{F_{j}}{2}+D I_{d}\right) z(j)+\left(\frac{F_{j+1}}{2}-D I_{d}\right) z(j+1)=\left(\frac{F_{j-1}}{2}+D I_{d}\right) z(j-1)+\left(\frac{F_{j}}{2}-D I_{d}\right) z(j)
$$

noting however that $z_{p^{+}-m}+(j)$ tends to 0 as $j$ tends to $+\infty$ and $z_{d+p^{-}+m}(j)$ tends to 0 as $j$ tends to $-\infty$. Thus, making the sum from $j$ to $+\infty$, we get

$$
\Delta z_{p^{+}-m}(j)=M_{j} z_{p^{+-m}}(j)-2\left(2 D I_{d}-F_{j+1}\right)^{-1} \sum_{l=j+1}^{+\infty} \Psi_{m}(l)
$$

and similarly

$$
\Delta z_{d+p^{-}+m}(j)=M_{j} z_{d+p^{-}+m}(j)+C_{j}^{-1} \sum_{l=-\infty}^{j} \Psi_{m}(l)
$$


Plugging (44), (43), (50) and (51) in the expression (48) of $\partial^{\varpi+1} \mathcal{D} / \partial \lambda^{\varpi+1}$, we get, performing elementary matrix manipulations,

$$
\begin{gathered}
\frac{\partial^{\varpi+1} \mathcal{D}}{\partial \lambda^{\varpi+1}}(0, j)=\operatorname{det}\left[\begin{array}{ccccccc}
\Phi_{1}^{\mathrm{I}}(0, j) & \ldots & \Phi_{p^{+}-\varpi-1}^{\mathrm{I}}(0, j) & \Psi_{p^{+-\varpi}}(j) & \ldots & \Psi_{0}(j) \\
0 & \ldots & & 0 & 0 & \ldots & 0 \\
r_{p^{+}+1}^{+} & \ldots & r_{d}^{+} & r_{1}^{-} & \ldots & r_{p^{-}-1}^{-} \\
-M_{j} r_{p^{+}+1}^{+} & \ldots & -M_{j} r_{d}^{+} & -M_{j} r_{1}^{-} & \ldots & -M_{j} r_{p^{-}-1}^{+} \\
z_{d+p^{-}}(j)-z_{p^{+}}(j) & & \ldots & z_{d+p^{-}+\varpi}(j)-z_{p^{+}-\varpi}(j) \\
2\left(2 D I_{d}-F_{j+1}\right)^{-1} \sum_{l \in \mathbb{Z}} \Psi_{0}(l) & \ldots & 2\left(2 D I_{d}-F_{j+1}\right)^{-1} \sum_{l \in \mathbb{Z}} \Psi_{\varpi}(l) \\
\Phi_{d+p^{-}+\varpi+1}^{\mathrm{I}}(0, j) & \ldots & \Phi_{2 d}^{\mathrm{I}}(0, j) \\
0 & \ldots & 0
\end{array}\right] .
\end{gathered}
$$

Changing the order of the columns in the determinant, we obtain a block determinant and the claimed equality.

Remark 2.2. Note that this expression is analogous to the expression one finds in the continuous case $[2,6,10]$.

Since the Evans function is analytic in a neighborhood of $\lambda=0$, assumption (H8) implies the existence of a positive $\sigma$ such that

$$
\text { the Evans function } \mathcal{D}(\cdot, j) \text { does not vanish in } D(0, \sigma) \backslash\{0\} \text {. }
$$

The continuity of the Evans function in $\Omega$ and assumption (H7) imply that there exists a positive $\eta$ such that

$$
\mathcal{D}(\cdot, j) \text { does not vanish in }\{\lambda \in \mathbb{C} / \operatorname{Re}(\lambda) \geq-\eta,|\operatorname{Im}(\lambda)| \leq \pi\} .
$$

We choose a small enough $\eta$ so that $\{\operatorname{Re}(\lambda)=-\eta\}$ intersects $\partial D(0, \sigma)$ outside $\mathcal{E}$ (see Fig. 3). We thus define the region

$$
\Omega^{\prime}:=\Omega \cap\{\lambda \in \mathbb{C} /-\eta \leq \operatorname{Re}(\lambda) \leq 1,|\operatorname{Im}(\lambda)| \leq \pi\}
$$

in which $\mathcal{D}$ is analytic and does not vanish.

\section{Estimates ON $\mathrm{G}_{\lambda}$}

Let $\lambda \in \Omega^{\prime} \backslash\{0\}, l \in \mathbb{Z}$ and $\vec{e} \in \mathbb{C}^{d}$. The Green's function $j \mapsto \mathrm{G}_{\lambda}(l, j) . \vec{e}$ of $(9)$ is the solution of

$$
\begin{aligned}
&\left(L-\mathrm{e}^{\lambda}\right) \mathrm{G}_{\lambda}(l, j) \cdot \vec{e}=-\delta_{l j} \mathrm{e}^{\lambda} \vec{e}, j \in \mathbb{Z}, \\
& \mathrm{G}_{\lambda}(l, j) \underset{j \rightarrow \pm \infty}{\longrightarrow} 0
\end{aligned}
$$

where $\delta$ is the Kronecker symbol. We want to compute estimates on

$$
\left(\begin{array}{c}
\mathrm{G}_{\lambda}(l, j) \\
\Delta_{j} \mathrm{G}_{\lambda}(l, j)
\end{array}\right)
$$

where $\Delta_{j} \mathrm{G}_{\lambda}(\cdot, j):=\mathrm{G}_{\lambda}(\cdot, j)-\mathrm{G}_{\lambda}(\cdot, j-1)$. 


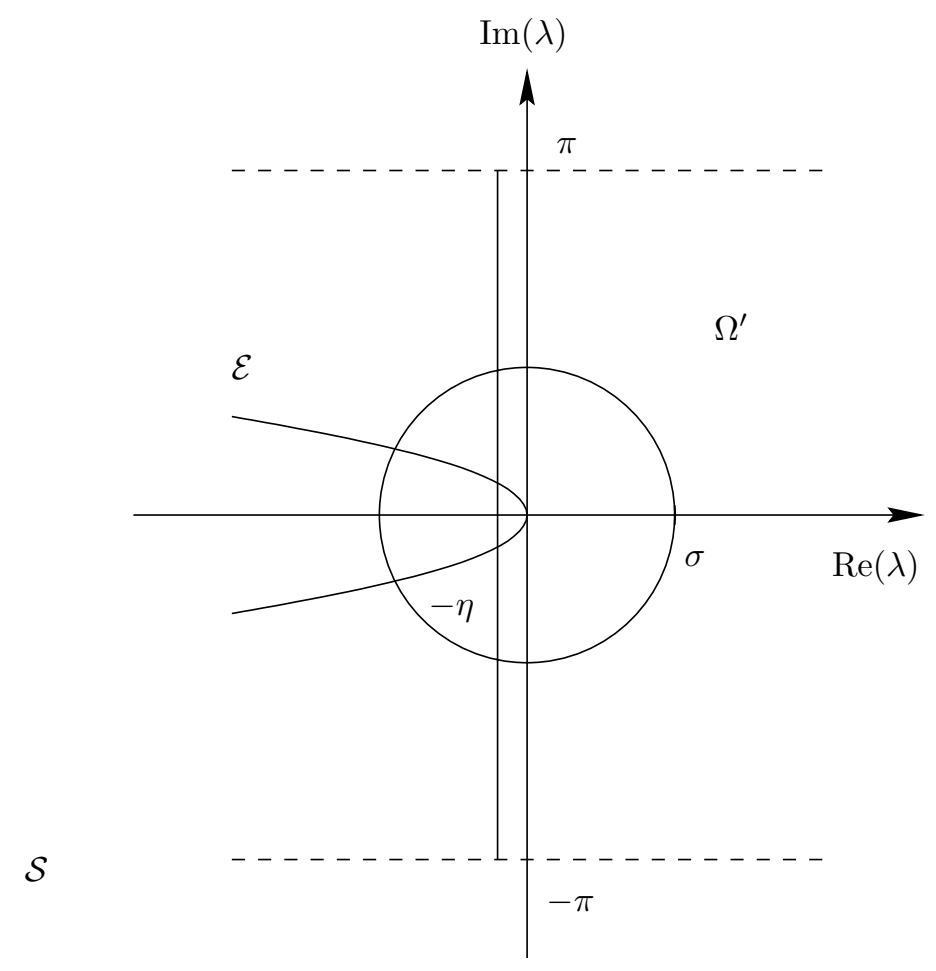

Figure 3. Definition of $\Omega^{\prime}$.

Since $\mathrm{G}_{\lambda}(l, \cdot)$ tends to 0 as $j$ tends to $\pm \infty$, we want to express $\mathrm{G}_{\lambda}(l, j)$ at $j \geq l$ (resp. at $j \leq l$ ) in an appropriate basis of the vector space of solutions of $L-\lambda=0$ that tends to 0 as $j$ tends to $+\infty$ (resp. as $j$ tends to $-\infty)$.

Proposition 3.1. For $\Omega^{\prime} \backslash\{0\}, \ell \in \mathbb{N}, l \in\{-\ell, \ldots, \ell\}$, and $\vec{e} \in \mathbb{C}^{d}, \mathrm{G}_{\lambda}(l, \cdot) . \vec{e}$ satisfies

$$
\begin{aligned}
\left(\begin{array}{c}
\mathrm{G}_{\lambda}(l, j) \cdot \vec{e} \\
\Delta_{j} \mathrm{G}_{\lambda}(l, j) \cdot \vec{e}
\end{array}\right) & =\sum_{q=1}^{d}\left(\nu_{q}(\lambda, l) \cdot \vec{e}\right) W_{q}(\lambda, j), \quad j \geq l, \\
& =\sum_{q=d+1}^{2 d}\left(\nu_{q}(\lambda, l) \cdot \vec{e}\right) W_{q}(\lambda, j), \quad j \leq l
\end{aligned}
$$

where $\left(W_{q}(\lambda, \cdot)\right)_{q \in\{1, \ldots, d\}}$ (resp. $\left.\left(W_{q}(\lambda, \cdot)\right)_{q \in\{d+1, \ldots, 2 d\}}\right)$ is an analytic basis of $E(\lambda)$ (see Eq. (39)) (resp. a basis of $F(\lambda)$ (see Eq. (40))), such that $W_{q}(\lambda, j)=V_{q}(\lambda, j) \mathrm{w}_{q}^{j}(\lambda)$ (see Lem. 3.1 for notations), $\nu_{q}(\lambda, l): \mathbb{R}^{d} \rightarrow \mathbb{R}$ is a linear form which is holomorphic in $D(0, \sigma)$ if $\varpi=-1$ or if $q \in\{1, \ldots, 2 d\} \backslash\left(\Upsilon^{+} \cup \Upsilon w^{-}\right)$, with $\Upsilon^{+}:=$ $\left\{p^{+}-\varpi, \ldots, p^{+}\right\}$and $\Upsilon^{-}:=\left\{d+p^{-}, \ldots, d+p^{-}+\varpi\right\}$; and meromorphic with a pole of order 1 at $\lambda=0$ otherwise, such that

$$
\left\|\nu_{q}(\lambda, l)\right\| \leq \frac{C(\ell)}{|\lambda|}, q \in \Upsilon^{+} \cup \Upsilon^{-}, \varpi \geq 0 .
$$


Moreover, with the notations of Proposition 2.1, the residual term $\mathcal{R}_{0}(l, j)$ that appears in Theorem 1.1 is the residue of $\mathrm{G}_{\lambda}$ at $\lambda=0$ and, for $l, j \in \mathbb{Z}$, it is given by the formula

$$
\begin{aligned}
\mathcal{R}_{0}(l, j): & \mathbb{C}^{d} \longrightarrow \mathbb{C}^{d} \\
& \vec{e} \mapsto \chi_{\varpi \geq 0} \sum_{m=0}^{\varpi} \frac{\phi_{l}^{\varpi}\left(S_{0}, \ldots, S_{m-1}, \vec{e}, S_{m+1}, \ldots, S_{\varpi}\right)}{\phi_{l}^{\varpi}\left(S_{0}, \ldots, S_{\varpi}\right)} \Psi_{m}(j),
\end{aligned}
$$

Proof. Let us find an appropriate basis of the vector spaces $E(\lambda)$ and $F(\lambda)$. In order to find a basis of $E(\lambda)$ that matches the behavior of the solutions we considered in Section 2 at $\lambda=0$, we prove the following lemma:

Lemma 3.1. Let $l \in \mathbb{Z}, \mathbf{V}(\lambda)$ be an eigenvector of $\mathbb{A}^{+}(\lambda)$ associated with $\mathrm{w}(\lambda)$ that never vanishes. Assuming that $\mathrm{w}, \mathbf{V}$ are analytic and that there exists $\omega \in(0,1)$ such that $\left|\mathrm{w}^{-1}(\lambda)\left(\mathbb{A}_{j}(\lambda)-\mathbb{A}^{+}(\lambda)\right)\right|=O\left(\omega^{j}\right)$ as $j$ tends to $+\infty$, there exists a solution $W(\lambda, j)$ of

$$
W_{j}=\mathbb{A}_{j}(\lambda) W_{j-1}
$$

such that $W(\lambda, j)=V(\lambda, j) \mathrm{w}^{j}(\lambda)$, where $V(\lambda, j)$ is analytic with respect to $\lambda$ and satisfies

$$
\forall m \geq 0, \forall j \geq l, \frac{\partial^{m} V}{\partial \lambda^{m}}(\lambda, j)=\frac{\partial^{m} \mathbf{V}}{\partial \lambda^{m}}(\lambda)+O\left(\omega^{j}\right)
$$

Remark 3.1. This lemma is a simplified (discrete) version of Zumbrun and Howard's Proposition 3.1 [27].

Proof. We set $l=0$. Let $W_{j}=V_{j} \mathrm{w}^{j}(\lambda)$ be a solution of $W_{j}=\mathbb{A}_{j}(\lambda) W_{j-1}$. The sequence $\left(V_{j}\right)_{j}$ satisfies the recurrence

$$
V_{j}=\mathrm{w}^{-1}(\lambda) \mathbb{A}_{j}(\lambda) V_{j-1}
$$

that we rewrite as

$$
V_{j}=\mathrm{w}^{-1}(\lambda) \mathbb{A}^{+}(\lambda) V_{j-1}+\mathrm{w}^{-1}(\lambda)\left(\mathbb{A}_{j}(\lambda)-\mathbb{A}^{+}\right) V_{j-1}
$$

We search a sequence $(V(\lambda, j))_{j}$ such that $V(\lambda, j) \rightarrow \mathbf{V}(\lambda)$ as $j$ tends to $+\infty$.

In a neighborhood of a fixed $\lambda_{0}$, we define the projection $P(\lambda)$ (resp. $Q(\lambda)$ ) on the direct sum of the eigenspaces associated with the eigenvalues of $\mathbb{A}^{+}(\lambda)$ of modulus strictly smaller than $|w(\lambda)|$ (resp. larger or equal to $|\mathrm{w}(\lambda)|)$ such that $P(\lambda)$ and $Q(\lambda)$ are complementary.

The projections $P(\lambda)$ and $Q(\lambda)$ are analytic in a neighborhood of $\lambda_{0}$ by classical matrix perturbation theory [15]. We also have the following estimates

$$
\begin{aligned}
& \left|\mathrm{w}^{-j}(\lambda)\left(\mathbb{A}^{+}(\lambda)\right)^{j} P(\lambda)\right| \leq \theta^{j}, \forall j \in \mathbb{N}, \\
& \left|\mathrm{w}^{-j}(\lambda)\left(\mathbb{A}^{+}(\lambda)\right)^{j} Q(\lambda)\right| \leq 1, \forall j \in \mathbb{N},
\end{aligned}
$$

where $\theta \in(0,1)$ is defined by $\theta=\min \left\{|\tilde{\mathrm{w}} / \mathrm{w}(\lambda)|, \tilde{\mathrm{w}} \in \sigma\left(\mathbb{A}^{+}(\lambda)\right)\right.$ and $\left.\tilde{\mathrm{w}}<\mathrm{w}(\lambda)\right\}$. Let $J \in \mathbb{Z}$. We define the map $T$ on $\ell^{\infty}(\{J, \ldots,+\infty\})$ by

$$
\begin{aligned}
(T V)_{j}= & \mathbf{V}(\lambda)+\sum_{l=J}^{j} \mathrm{w}^{j-l}(\lambda)\left(\mathbb{A}^{+}(\lambda)\right)^{j-l} P(\lambda) \mathrm{w}^{-1}(\lambda)\left(\mathbb{A}_{l-1}(\lambda)-\mathbb{A}^{+}(\lambda)\right) V_{l-1} \\
& -\sum_{l=j+1}^{+\infty} \mathrm{w}^{j-l}(\lambda)\left(\mathbb{A}^{+}(\lambda)\right)^{j-l} Q(\lambda) \mathrm{w}^{-1}(\lambda)\left(\mathbb{A}_{l-1}(\lambda)-\mathbb{A}^{+}(\lambda)\right) V_{l-1} .
\end{aligned}
$$


Let $V_{1}$ and $V_{2}$ be two sequences. By using (61) and (62), we have

$$
\begin{aligned}
\left|T V_{1}-T V_{2}\right|_{j} & \leq \sum_{l=J}^{j} \theta^{j-l} \omega^{l-1}\left|V_{1}-V_{2}\right|_{\infty}+\sum_{l=j+1}^{+\infty} \omega^{l-1}\left|V_{1}-V_{2}\right|_{\infty} \\
& \leq\left(\sum_{l=J}^{j} \theta^{j-l} \omega^{l-1}+\sum_{j+1}^{+\infty} \omega^{l-1}\right)\left|V_{1}-V_{2}\right|_{\infty} \\
& \leq C \omega^{J}\left|V_{1}-V_{2}\right|_{\infty},
\end{aligned}
$$

where $C$ is a positive constant that does not depend on $j$.

By choosing a large enough $J$, we can set

$$
C \omega^{J} \leq \frac{1}{2}
$$

so that the map $\mathcal{T}$ is a contraction of $\ell^{\infty}(\{J, \ldots,+\infty\})$. Iterating $\mathcal{T}$ on $V_{0}=0$, we obtain a solution $V \in$ $\ell^{\infty}(\{J, \ldots,+\infty\})$ of the equation $\mathcal{T} V=V$. Computing $V_{j+1}$ shows that $V$ is a bounded solution of $\mathcal{T} V=V$ if and only if $V$ is a bounded solution of (60). We conclude by noting that $\mathcal{T}$ preserves analyticity in $\lambda$.

A similar result holds for the eigenvalues and eigenvectors of $\mathbb{A}^{-}(\lambda)$.

Next, we prove that, if $\eta$ (see Eq. (53)) is small enough, the eigenvalues of $\mathbb{A}^{ \pm}(\lambda)$ remain simple for all $\lambda \in \Omega^{\prime} \backslash\{0\}$, so that, considering the set of sequences $\left(W_{q}(\lambda, \cdot)\right)_{q \in\{1, \ldots, d\}}\left(\operatorname{resp} .\left(W_{q}(\lambda, \cdot)\right)_{q \in\{d+1, \ldots, 2 d\}}\right)$ given by Lemma 3.1 for each eigenvalue of $\mathbb{A}^{+}(\lambda)$ (resp. of $\mathbb{A}^{-}(\lambda)$ ) of modulus strictly smaller than 1 (resp. of modulus strictly larger than 1 ), we obtain a basis of $E(\lambda)$ (resp. of $F(\lambda)$ ).

Lemma 3.2. Let $\lambda \in \Omega^{\prime} \backslash\{0\}$. The characteristic polynomial $\Pi^{ \pm}(\cdot, \lambda)$ of $\mathbb{A}^{ \pm}(\lambda)$ has no double root.

Proof. Recalling the expression of $\Pi^{ \pm}$given by (36), we rewrite it as $\Pi^{ \pm}=(2 D+a)^{-d} \prod_{q \in\{1, \ldots, d\}} \Pi_{q}^{ \pm}$, with

$$
\Pi_{q}^{ \pm}:=\left(2 D+a_{q}^{ \pm}\right) \mathrm{w}^{2}+2\left(1-2 D-\mathrm{e}^{\lambda}\right) \mathrm{w}+2 D-a_{q}^{ \pm}=0
$$

For the sake of simplicity, we drop the superscript \pm .

Let $w$ be a root of $\Pi$ with a strictly larger than 1 multiplicity. We must consider two cases:

case 1: there exists $q \in\{1, \ldots, d\}$ such that $\mathrm{w}$ is a double root of $\Pi_{q}$,

case 2: there exist $q_{1}, q_{2}$ in $\{1, \ldots, d\}, q_{1} \neq q_{2}$, such that $w$ is a root of both $\Pi_{q_{1}}$ and $\Pi_{q_{2}}$.

Let us deal at first with case 1:

if $\mathrm{w}$ is a double root of $\Pi_{q}$, then the discriminant of $\Pi_{q}$ vanishes, that is

$$
\left(1-2 D-\mathrm{e}^{\lambda}\right)^{2}=4 D^{2}-a_{q}^{2}
$$

which is equivalent to

$$
\left(1-\mathrm{e}^{\lambda}\right)^{2}-4 D\left(1-\mathrm{e}^{\lambda}\right)+a_{q}^{2}=0
$$

Denoting $s:=1-\mathrm{e}^{\lambda}$ and splitting (63) into real and imaginary parts, we obtain the following system

$$
\begin{aligned}
\operatorname{Re}(s)^{2}-\operatorname{Im}(s)^{2}-4 D \operatorname{Re}(s)+a_{q}^{2} & =0 \\
(\operatorname{Re}(s)-2 D) \operatorname{Im}(s) & =0
\end{aligned}
$$


so that, considering (65), either $\operatorname{Im}(s)=0$ and $\operatorname{Re}(s)=2 D \pm \sqrt{4 D^{2}-a_{q}^{2}}$, that is $\lambda \leq \Lambda$ (see Eq. (37)), and $\lambda$ does not belong to $\Omega^{\prime}$, or $\operatorname{Im}(s) \neq 0$ and $\operatorname{Re}(s)=2 D$ : plugging the value of $\operatorname{Re}(s)$ in $(64)$, we get $\operatorname{Im}(s)= \pm a_{q}$, that is $\mathrm{e}^{\lambda}=1-2 D \pm a_{q}$. Using the CFL condition (H5), we show that the modulus of $\mathrm{e}^{\lambda}$ is strictly less than 1 :

$$
\begin{aligned}
\left|\mathrm{e}^{\lambda}\right|^{2}-1 & =(1-2 D)^{2}+a_{q}^{2}-1 \\
& =4 D^{2}-4 D+a_{q}^{2} \\
& <8 D^{2}-4 D \\
& <0 .
\end{aligned}
$$

Consequently, choosing a small enough $\eta, \lambda$ does not belong to $\Omega^{\prime}$.

Secondly, we consider case 2 :

if $\mathrm{w}$ solves both $\Pi_{q_{1}}=0$ and $\Pi_{q_{2}}=0$, it also solves the equation $\Pi_{q_{1}}-\Pi_{q_{2}}=0$ :

$$
\left(a_{q_{1}}-a_{q_{2}}\right) \mathrm{w}^{2}+\left(a_{q_{2}}-a_{q_{1}}\right)=0 .
$$

Since the eigenvalues of $\mathrm{d} f\left(u^{ \pm}\right)$are real and simple (H2), w necessarily satisfies $\mathrm{w}^{2}-1=0$. But, by definition, $\Omega^{\prime}$ does not contain any $\lambda$ such that the modulus of corresponding eigenvalues of $\mathbb{A}^{ \pm}(\lambda)$ is 1 .

Let $l \in \mathbb{Z}$. Comparing the behaviors of $\left(W_{q}\right)$ (see Eq. (59)) and of the sequences $\left(\Phi_{q}\right)$ that were defined in Section 2.2 (see Eq. (41)), and reordering the indices appropriately, we can take

$$
\begin{aligned}
& \Phi_{q}(\lambda, j) \underset{\lambda \rightarrow 0}{=} W_{q}(\lambda, j), j \geq l, q \in\{1, \ldots, d\} \\
& \Phi_{q}(\lambda, j) \underset{\lambda \rightarrow 0}{=} W_{q}(\lambda, j), j \leq l, q \in\{d+1, \ldots, 2 d\} .
\end{aligned}
$$

From now on, we only keep the " $\Phi$ " notation.

Let $l \in\{-\ell, \ldots, \ell\}, j \in \mathbb{Z}$ and $\vec{e} \in \mathbb{C}^{d}$. Knowing that $\mathrm{G}_{\lambda}(l, j) . \vec{e}$ solves (54), that is $\mathrm{G}_{\lambda}(l, j) . \vec{e}$ satisfies

$$
\left(D I_{d}+\frac{F_{j-1}}{2}\right) \mathrm{G}_{\lambda}(l, j-1) \cdot \vec{e}+\left(1-2 D-\mathrm{e}^{\lambda}\right) \mathrm{G}_{\lambda}(l, j) \cdot \vec{e}+\left(D I_{d}-\frac{F_{j+1}}{2}\right) \mathrm{G}_{\lambda}(l, j+1) \cdot \vec{e}=-\delta_{j l} \mathrm{e}^{\lambda} \vec{e}, j, l \in \mathbb{Z},
$$

we search $\mathrm{G}_{\lambda}(l, j) \cdot \vec{e}$ of $(66)$ in the form

$$
\begin{aligned}
\mathrm{G}_{\lambda}(l, j) \cdot \vec{e} & =\sum_{q=1}^{d} \nu_{q}(\lambda, l) \cdot \vec{e} \Phi_{q}^{\mathrm{I}}(\lambda, j), \quad \text { if } j \geq l, \\
& =\sum_{q=d+1}^{2 d} \nu_{q}(\lambda, l) \cdot \vec{e} \Phi_{q}^{\mathrm{I}}(\lambda, j), \quad \text { if } j \leq l,
\end{aligned}
$$

where $\nu_{q}(\lambda, l) \in \mathbb{C}$.

Taking equation (66) at $j=l$, we have:

$$
\begin{aligned}
& \sum_{q=1}^{d} \nu_{q}(\lambda, l) \cdot \vec{e} \Phi_{q}^{\mathrm{I}}(\lambda, l)=\sum_{q=d+1}^{2 d} \nu_{q}(\lambda, l) \cdot \vec{e} \Phi_{q}^{\mathrm{I}}(\lambda, l), \\
& \sum_{q=1}^{d} \nu_{q}(\lambda, l) \cdot \vec{e} \Phi_{q}^{\mathrm{II}}(\lambda, l)=\sum_{q=d+1}^{2 d} \nu_{q}(\lambda, l) \cdot \vec{e} \Phi_{q}^{\mathrm{II}}(\lambda, l)+2\left(2 D I_{d}-F_{l+1}\right)^{-1} \mathrm{e}^{\lambda} \vec{e} .
\end{aligned}
$$


Let $\mathcal{G}(\lambda, l):=\left(\Phi_{1}(\lambda, l), \ldots, \Phi_{2 d}(\lambda, l)\right)$. Its determinant is $\mathcal{D}(\lambda, l)$ (see Eq. (45)). The assumption (H8) gives

$$
\left|\mathcal{D}(\lambda, l)^{-1}\right| \leq \frac{C(l)}{|\lambda|^{\varpi+1}}
$$

locally in $\lambda$ and in $l$, that is, if $\varpi=-1, \mathcal{G}(\cdot, l)^{-1}$ is holomorphic in $\Omega^{\prime}$; otherwise, $\mathcal{G}(\lambda, l)^{-1}$ is meromorphic for $\lambda \in \Omega^{\prime}, \lambda=0$ being a pole of order at most $\varpi+1$ because of (H8). Consequently, the linear forms $\left(\nu_{q}(\lambda, l)\right)$ are also holomorphic in $\Omega^{\prime}$ if $\varpi=-1$ and meromorphic with a pole at $\lambda=0$ if $\varpi \geq 0$.

Let us now examine the behavior of $\mathcal{G}(\lambda, l)$ and of $\left(\nu_{q}(\lambda, l)\right)$ as $\lambda$ tends to 0 when $\varpi \geq 0$ to determine the order of the pole.

Let $\lambda \in \Omega^{\prime} \backslash\{0\}$. We have

$$
\left(\nu_{1}(\lambda, l), \ldots, \nu_{d}(\lambda, l),-\nu_{d+1}(\lambda, l), \ldots,-\nu_{2 d}(\lambda, l)\right)^{T}=\mathcal{G}(\lambda, l)^{-1}\left(0,2\left(2 D I_{d}-F_{l+1}\right)^{-1} \vec{e}\right)^{T}
$$

Denote by $\operatorname{com}(\mathcal{G})(\lambda, l)$ the comatrix of $\mathcal{G}(\lambda, l)$. For $\lambda \in \Omega^{\prime}$, we have

$$
\mathcal{G}(\lambda, l) \operatorname{com}(\mathcal{G})^{T}(\lambda, l)=\mathcal{D}(\lambda, l) I_{d}
$$

Applying Leibniz's formula and recalling (H8), the $\varpi$ th derivative of (72) reads

$$
\begin{aligned}
\sum_{m=0}^{\varpi}\left(\begin{array}{c}
\varpi \\
m
\end{array}\right) \frac{\partial^{\varpi-m} \mathcal{G}}{\partial \lambda^{\varpi-m}}(\lambda, l) \frac{\partial^{m} \operatorname{com}(\mathcal{G})^{T}}{\partial \lambda^{m}}(\lambda, l) & =\frac{\partial^{\varpi} \mathcal{D}}{\partial \lambda^{\varpi}}(\lambda, l) I_{2 d} \\
& =\lambda \frac{\partial^{\varpi+1} \mathcal{D}}{\partial \lambda^{\varpi+1}}(0, l)+o(|\lambda|) .
\end{aligned}
$$

Let $\operatorname{com}(\mathcal{G})(\lambda, l)=:\left((-1)^{q+q^{\prime}} g_{q q^{\prime}}(\lambda, l)\right)_{1 \leq q, q^{\prime} \leq 2 d}$. The coefficient $g_{q q^{\prime}}(\lambda, l)$ is a determinant of order $2 d-1$ that we obtain by removing the $q$ th line and the $q^{\prime}$ th column of $\operatorname{det}(\mathcal{G}(\lambda, l))$.

Consequently, $\left(g_{q q^{\prime}}(\lambda, l)\right)$ are polynomial in the coefficients of $\mathcal{G}(\lambda, l)$ so that $\left(g_{q q^{\prime}}(\lambda, l)\right)$ tend to finite limits as $\lambda$ tends to 0 .

If $m \in\{0, \ldots, \varpi-1\}$, a similar computation as the one carried out in Section 2.1 yields

$$
\frac{\partial^{m} g_{q q^{\prime}}}{\partial \lambda^{m}}(0, l)=0, \forall q, q^{\prime} \in\{1, \ldots, 2 d\}
$$

So equation $(73)$ reduces to

$$
\lambda \mathcal{G}^{-1}(\lambda, l) \underset{\lambda \rightarrow 0}{\longrightarrow} \frac{\frac{\partial^{\varpi} \operatorname{com}(\mathcal{G})^{T}}{\partial \lambda^{\varpi}}(0, l)}{\frac{\partial^{\varpi+1} \mathcal{D}}{\partial \lambda^{\varpi+1}}(0, l)}
$$

Moreover, taking the derivative of $\operatorname{com}(\mathcal{G}(\lambda, l))$ a step further, if $q^{\prime} \in\{1, \ldots, 2 d\} \backslash\left(\Upsilon^{+} \cup \Upsilon^{-}\right)$, with $\Upsilon^{+}=$ $\left\{p^{+}-\varpi, \ldots, p^{+}\right\}$and $\Upsilon^{-}=\left\{d+p^{-}, \ldots, d+p^{-}+\varpi\right\}$, we have

$$
\frac{\partial^{\varpi} g_{q q^{\prime}}}{\partial \lambda^{\varpi}}(0, l)=0
$$

because of our choice (46) of $\Psi$ (see the computation of the $(\varpi+1)$ th derivative of $\mathcal{D}$ at $\lambda=0$ in Sect. 2.1 for details). 
Thus, on the one hand, for $q \in\{1, \ldots, 2 d\} \backslash\left(\Upsilon^{+} \cup \Upsilon^{-}\right)$, we get

$$
\lim _{\lambda \rightarrow 0} \lambda \nu_{q}(\lambda, l)=0
$$

that is $\nu_{q}(\cdot, l)$ is in fact holomorphic in $\Omega^{\prime}$ for $q \in\{1, \ldots, 2 d\} \backslash\left(\Upsilon^{+} \cup \Upsilon^{-}\right)$.

On the other hand, for $q \in \Upsilon^{ \pm}$, we obtain

$$
\lim _{\lambda \rightarrow 0} \lambda \nu_{q}(\lambda, l)= \pm \frac{\frac{\partial^{\varpi} \widehat{\mathcal{D}}_{q}(0, l)}{\partial \lambda^{\varpi}}}{\frac{\partial^{\varpi+1} \mathcal{D}(0, l)}{\partial \lambda^{\varpi+1}}},
$$

where

$$
\widehat{\mathcal{D}}_{q}(\lambda, j):=\operatorname{det}\left(\Phi_{1}(0, l), \ldots, \Phi_{q-1}(0, l),\left(\begin{array}{c}
0 \\
2\left(2 D I_{d}-F_{l+1}\right)^{-1} \vec{e}
\end{array}\right), \Phi_{q+1}(0, l), \ldots, \Phi_{2 d}(0, l)\right)
$$

The same computation as for the $(\varpi+1)$ th derivative of $\mathcal{D}$ yields, for $q \in \Upsilon^{ \pm}, m \in\{0, \ldots, \varpi\}$,

$$
\frac{\partial^{\varpi} \widehat{\mathcal{D}}_{q}}{\partial \lambda^{\varpi}}(0, l)= \pm(-1)^{p^{-}+1} \operatorname{det}\left(M_{l}\right) \mathcal{W}(l) \phi_{l}^{\varpi}\left(S_{0}, \ldots, S_{m-1}, \vec{e}, S_{m+1}, \ldots, S_{\varpi}\right)
$$

so that, recalling the expression $(47)$ of $\left(\partial^{\varpi+1} \mathcal{D}(\lambda, l) / \partial \lambda^{\varpi+1}\right)$, we finally get the equality

$$
\lim _{\lambda \rightarrow 0} \lambda \nu_{d+p^{-}+m}(\lambda, l)=\lim _{\lambda \rightarrow 0} \lambda \nu_{p^{+-m}}(\lambda, l)=\frac{\phi_{l}^{\varpi}\left(S_{0}, \ldots, S_{m-1}, \vec{e}, S_{m+1}, \ldots, S_{\varpi}\right)}{\phi_{l}^{\varpi}\left(S_{0}, \ldots, S_{\varpi}\right)}, \forall m \in\{0, \ldots, \varpi\} .
$$

So the order of the pole of $\nu_{q}(\lambda, l)$, for $q \in\{1, \ldots, 2 d\}$, is 1 and (57) is proved.

Gathering (74) and (75), we have

$$
\lim _{\lambda \rightarrow 0} \lambda \mathrm{G}_{\lambda}(l, j) \cdot \vec{e}=\sum_{m=0}^{\varpi}\left(\frac{\phi_{l}^{\varpi}\left(S_{0}, \ldots, S_{m-1}, \vec{e}, S_{m+1}, \ldots, S_{\varpi}\right)}{\phi_{l}^{\varpi}\left(S_{0}, \ldots, S_{\varpi}\right)}\right) \Psi_{m}(j)=\mathcal{R}_{0}(l, j), \forall j \in \mathbb{Z} .
$$

Furthermore, a straightforward computation shows that the linear mapping $u=\left(u_{l}\right) \mapsto \sum_{l \in \mathbb{Z}} \mathcal{R}_{0}(l, \cdot) u_{l}$ is a projection on the kernel of $L-1$.

\section{Proof of Theorem 1.1}

We want to compute estimates on the Green's function of (9) by using an inverse Laplace transform

$$
\mathrm{G}(n, l, j)=\frac{1}{2 i \pi} \int_{\Gamma} \mathrm{e}^{\lambda n} \mathrm{G}_{\lambda}(l, j) \mathrm{d} \lambda .
$$

Recall that G solves

$$
\begin{aligned}
\mathrm{G}(n+1, l, j)-L \mathrm{G}(n, l, j) & =0, \text { for } n \geq 1 \\
\mathrm{G}(0, l, j) & =\delta_{l j} I_{d},
\end{aligned}
$$

so, since $L$ is a second-order recurrence operator, the speed of propagation is finite (see Fig. 4) and

$$
\mathrm{G}(n, l, j)=0 \text { for }|l-j| \geq n+1 \text {. }
$$




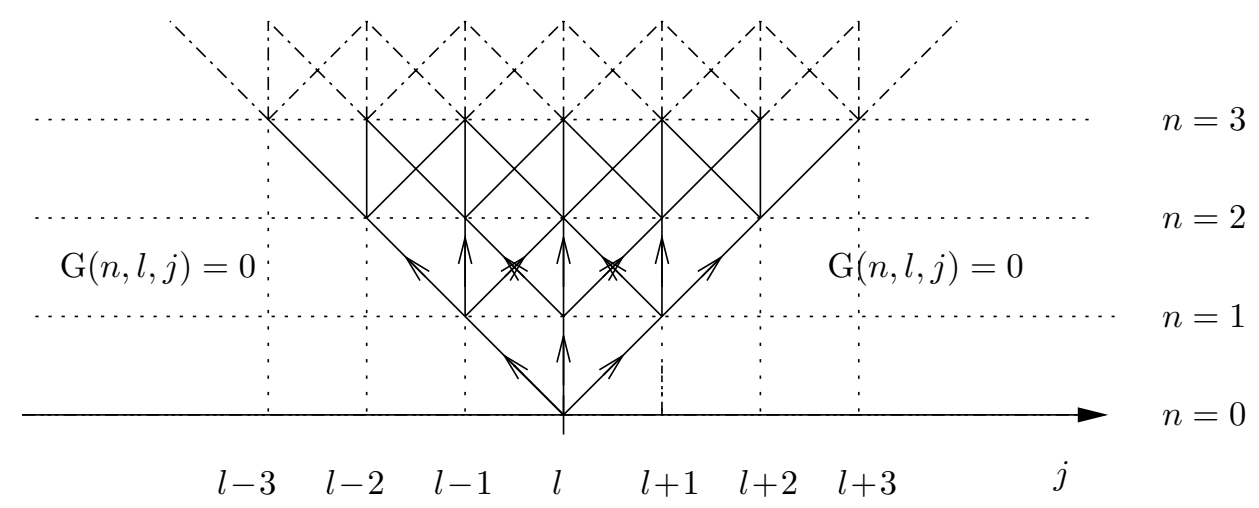

Figure 4. Propagation.

Since we assumed that the only pole of $\mathrm{G}_{\lambda}$ in $\{\lambda / \operatorname{Re}(\lambda) \geq-2 \eta\}$ is at $\lambda=0$, the initial path of integration in the formula of the inverse Laplace transform can be any segment $\Gamma:=[s-i \pi, s+i \pi]$ with $s>0$. However, thanks to the Cauchy formula, we can change this path to better suit the behavior of $\mathrm{G}_{\lambda}$, noting that if the pole $\lambda=0$ is inside the area enclosed in the closed path $\Gamma \cup S_{1} \cup \Gamma^{\prime} \cup S_{u}$, where $\Gamma^{\prime}$ is the new path of integration and $S_{1}$ and $S_{u}$ are the lower (resp. upper) segment that is enclosed in $\{\operatorname{Im}(\lambda)=-\pi\}$ (resp. $\left.\{\operatorname{Im}(\lambda)=\pi\}\right)$ that appears when we modify $\Gamma$ as in Figure 5 , the residue of $G_{\lambda}$ at $\lambda=0, \mathcal{R}_{0}$ (see Sect. 3.1), will appear in the formula (76).

Remark 4.1. Since the orientations of $\mathrm{S}_{\mathrm{l}}$ and $\mathrm{S}_{\mathrm{u}}$ are opposite and $\lambda \mapsto \mathrm{e}^{\lambda n} \mathrm{G}_{\lambda}(l, j)$ is $i 2 \pi$-periodic, the integrals along $S_{u}$ and $S_{l}$ compensate each other. Therefore, we will no longer mention them.

Let us now treat the case of medium $(l-j)^{2} / n^{2}$.

Proposition 4.1. There exists a positive constant $\beta$ such that, for all $\ell \in \mathbb{N}$, there exist $C(\ell)>0$ and $\tau(\ell)>0$ so that for all $l \in\{-\ell, \ldots, \ell\}, j \in \mathbb{Z}$ and $n \in \mathbb{N}$ satisfying

$$
\beta \sigma n^{2} \leq(l-j)^{2} \leq n^{2}
$$

the following estimate holds

$$
\left\|\begin{array}{c}
\mathrm{G}(n, l, j) \\
\Delta_{j} \mathrm{G}(n, l, j)
\end{array}\right\| \leq C(\ell) \exp \left(-\tau(\ell) \frac{(l-j)^{2}}{n}\right) .
$$

Proof. In the following, $C(\ell)$ will denote a generic constant depending on $\ell$. Let $\ell \in \mathbb{N}, l \in\{-\ell, \ldots, \ell\}$ and $j \in \mathbb{Z}$. Let

$$
\lambda_{m}:=\gamma(\ell)(l-j)^{2} /(\beta n)^{2}
$$

We will integrate on $\Gamma_{1}:=\Gamma_{1}^{-} \cup \Gamma_{1}^{m} \cup \Gamma_{1}^{+}$(see Fig. 6), where, assuming that $\eta$ is so small that $\gamma_{1}$ does not intersect $\mathcal{E}$,

$$
\begin{aligned}
& \Gamma_{1}^{-}:=\left\{\lambda=-\eta+i y,-\pi \leq y \leq-\eta-\lambda_{m}\right\}, \\
& \Gamma_{1}^{m}=\left\{|\operatorname{Im}(\lambda)|=\lambda_{m}-\operatorname{Re}(\lambda),-\eta \leq \operatorname{Re}(\lambda) \leq \lambda_{m}\right\}, \\
& \Gamma_{1}^{+}:=\left\{\lambda=-\eta+i y, \eta+\lambda_{m} \leq y \leq \pi\right\}
\end{aligned}
$$




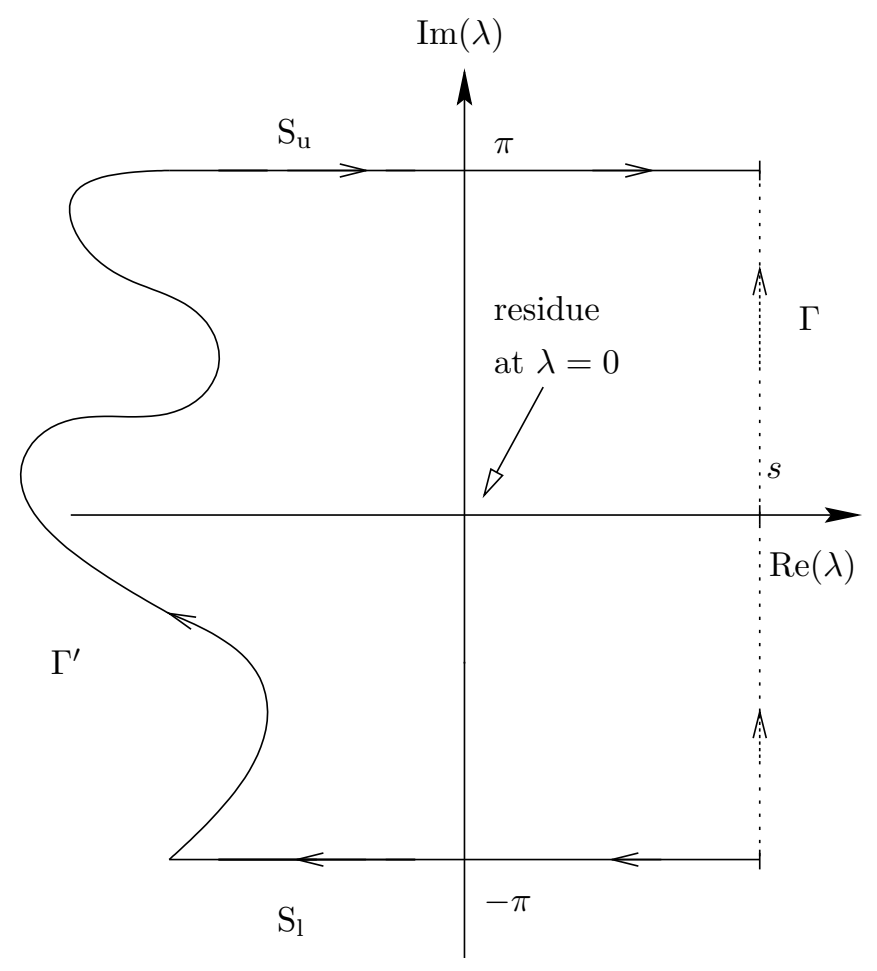

Figure 5. Path of integration $\Gamma$ and $\Gamma^{\prime}$.

We have here

$$
\mathrm{G}(n, l, j)=\frac{1}{2 i \pi}\left[\left(\int_{\Gamma_{1}^{-}}+\int_{\Gamma_{1}^{m}}+\int_{\Gamma_{1}^{+}}\right) \mathrm{e}^{\lambda n} \mathrm{G}_{\lambda}(l, j) \mathrm{d} \lambda\right]=I_{1}^{-}+I_{1}^{m}+I_{1}^{+}
$$

Using Proposition 3.1, we get at once

$$
\left|I_{1}^{ \pm}\right| \leq \frac{1}{2 \pi} \mathrm{e}^{-\eta n} C(\ell) \int_{\Gamma_{1}^{ \pm}} \frac{1}{|\lambda|}|\mathrm{d} \lambda|
$$

so, since $|\lambda| \geq \eta$ on $\Gamma_{1}^{ \pm}$, and $\left|\Gamma_{1}^{ \pm}\right| \leq \pi$

$$
\left|I_{1}^{-}\right|+\left|I_{1}^{+}\right| \leq \frac{C(\ell)}{\eta} \mathrm{e}^{-\eta n}
$$

We consider now $I_{1}^{m}$. Since $\operatorname{Re}(\lambda) \leq \lambda_{m}$ and $|\lambda| \geq \lambda_{m} \sqrt{2} \geq \gamma(\ell) \sigma$, we obtain, by applying Proposition 3.1,

$$
\begin{aligned}
\left|I_{1}^{m}\right| & \leq C(\ell) \mathrm{e}^{\lambda_{m} n-\gamma(\ell)|l-j|} \int_{\Gamma_{1}^{m}} \frac{1}{|\lambda|}|\mathrm{d} \lambda| \\
& \leq C(\ell) \frac{\sqrt{2}}{\sigma}\left|\Gamma_{1}^{m}\right| \mathrm{e}^{\lambda_{m} n-\gamma(\ell)|l-j|}
\end{aligned}
$$




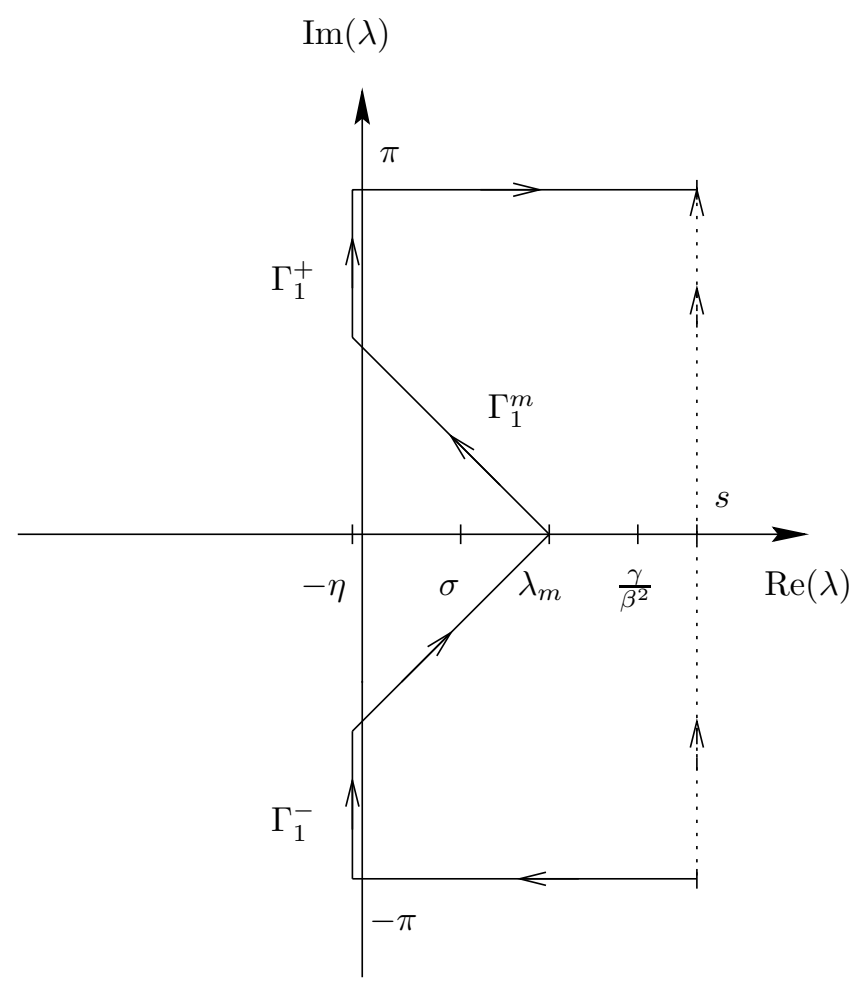

FiguRE 6. Path of integration $\Gamma_{1}$.

So, since $\left|\Gamma_{1}^{m}\right| \leq 2 \sqrt{2}\left(\lambda_{m}+\eta\right) \leq 2 \sqrt{2}(\gamma(\ell) / \beta+\eta) \leq C$ and $-|l-j| \leq-\frac{(l-j)^{2}}{n}$, we have

$$
\left|I_{1}^{m}\right| \leq C(\ell) \frac{\sqrt{2}}{\sigma} \exp \left(\gamma(\ell)(1-\beta) \frac{(l-j)^{2}}{\beta n}\right) .
$$

Since we can always assume that $\sigma<1$, we take $\beta \in(1,1 / \sigma)$.

The bounds on $\Delta_{j} \mathrm{G}(n, l, j)$ are obtained through the same computations.

We now have to consider the case of small $(l-j)^{2} / n^{2}$, that is $(l-j)^{2} \leq \sigma \beta n^{2}$.

Remark 4.2. Note that in this case, the following inequality holds

$$
\mathrm{e}^{-\eta n} \leq \mathrm{e}^{-\eta n / 2} \mathrm{e}^{-\eta(l-j)^{2} /(2 \sigma \beta n)}
$$

- Case $|\mathbf{l}-\mathbf{j}| \leq \ell$

Let us consider at first the case of bounded $n$ : we integrate $G_{\lambda}$ along $\Gamma_{2}:=\Gamma_{2}^{-} \cup \Gamma_{2}^{m} \cup \Gamma_{2}^{+}$(see Fig. 7), where

$$
\begin{aligned}
& \Gamma_{2}^{-}:=\{\lambda=-\eta+i y,-\pi \leq y \leq-\eta-\sigma\}, \\
& \Gamma_{2}^{m}=\{|\operatorname{Im}(\lambda)|=\sigma-\operatorname{Re}(\lambda),-\eta \leq \operatorname{Re}(\lambda) \leq \sigma\}, \\
& \Gamma_{2}^{+}:=\{\lambda=-\eta+i y, \eta+\sigma \leq y \leq \pi\} .
\end{aligned}
$$




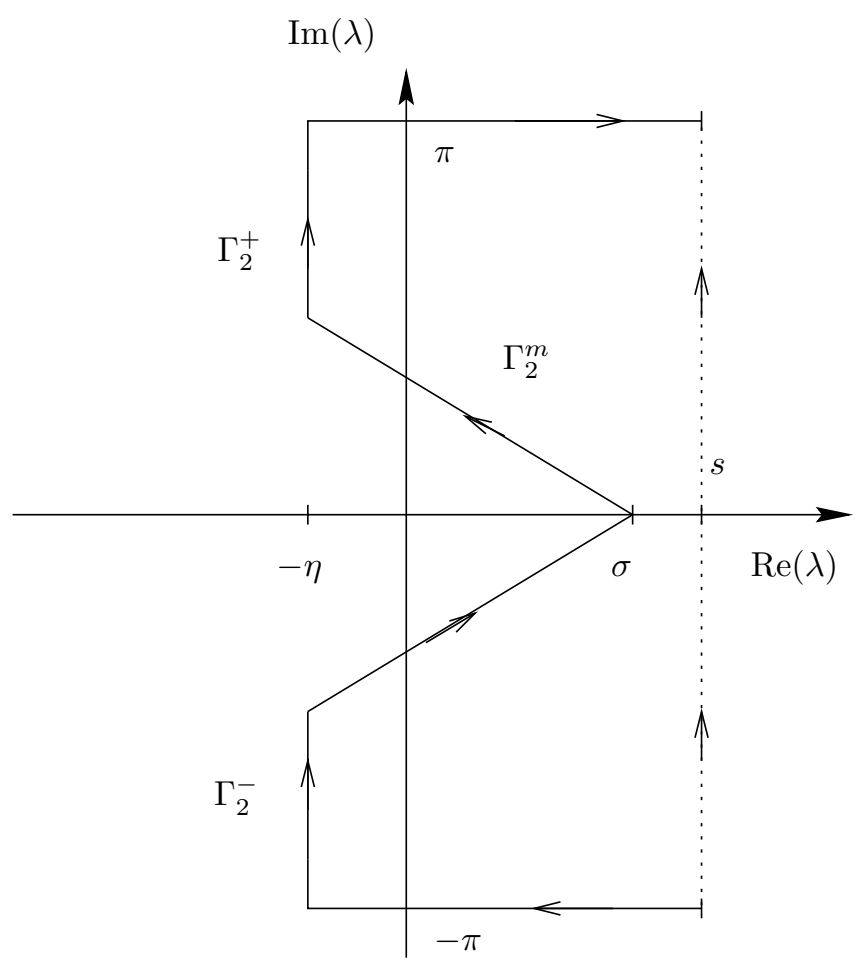

FiguRE 7. Path of integration $\Gamma_{2}$.

Using the same notations as in (80), we get at once, similarly as in (81),

$$
\left|I_{2}^{-}\right|+\left|I_{2}^{+}\right| \leq \frac{C(\ell)}{\eta}
$$

Since $\Gamma_{2}^{m} \subset D(0, \sigma) \backslash D(0, \sigma / \sqrt{2})$, we apply Proposition 3.1 and we get

$$
\left|I_{2}^{m}\right| \leq C(\ell) \frac{\sqrt{2}}{\sigma} \leq C(\ell) \mathrm{e}^{-\eta n}
$$

because $n$ is bounded. The claimed estimate follows from Remark 4.2.

Let us deal now with the case of large $n$ : we integrate $G_{\lambda}$ along $\Gamma_{0}$ (see Fig. 8). Define now $\Gamma_{0}:=\{\lambda=$ $-\eta+i y /-\pi \leq y \leq \pi\}$. We thus obtain

$$
\left|\mathrm{G}(n, l, j)-\mathcal{R}_{0}(l, j)\right| \leq \frac{C(\ell)}{2 \pi} \int_{-\pi}^{\pi} \mathrm{e}^{-\eta n} \mathrm{~d} y \leq C(\ell) \mathrm{e}^{-\eta n}
$$

We conclude by using once again Remark 4.2 . 


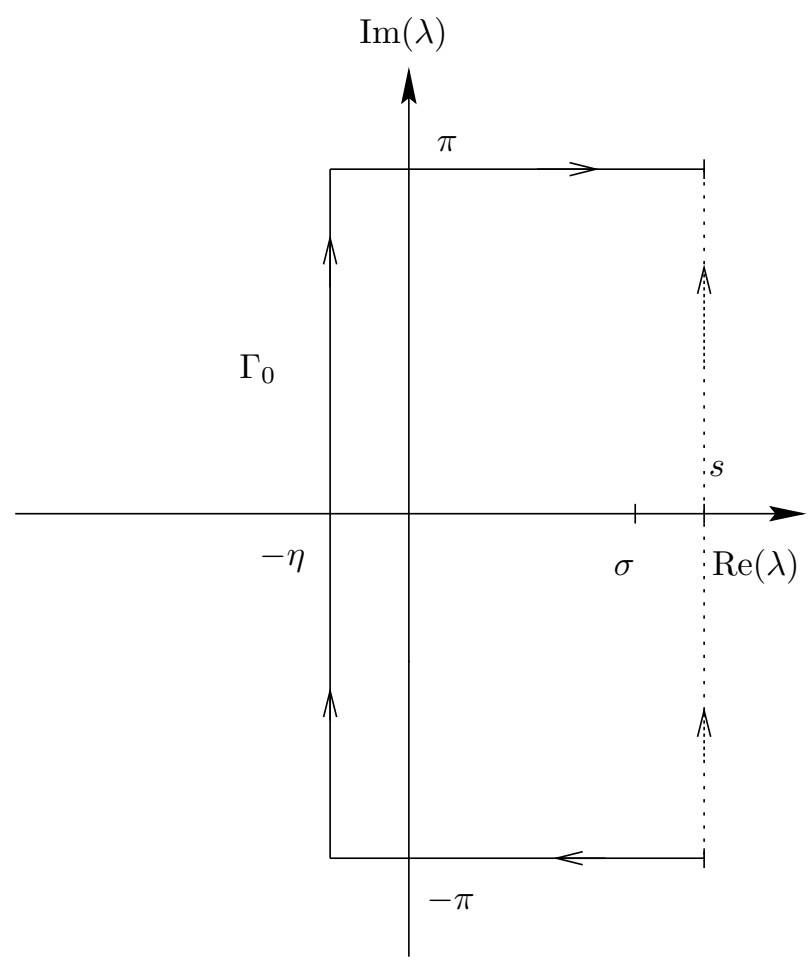

Figure 8. Path of integration $\Gamma_{0}$.

The computations are the same for the bounds on $\Delta_{j} \mathrm{G}(n, l, j)$;

- Case $|\mathbf{j}|>\ell \geq|\mathbf{l}|$

Thanks to Proposition 3.1, we know that

$$
\begin{aligned}
\int_{\Gamma} \mathrm{G}_{\lambda}(l, j) \mathrm{d} \lambda & =\sum_{q=1}^{d} \int_{\Gamma} \nu_{q}(\lambda, l) \Phi_{q}(\lambda, j), \forall j \geq l \\
& =\sum_{q=d+1}^{2 d} \int_{\Gamma} \nu_{q}(\lambda, l) \Phi_{q}(\lambda, j), \forall l \geq j
\end{aligned}
$$

for $\lambda \in D(0, \sigma)$, so that we need only estimate each of the $\left\|\nu_{q}(\lambda, l) \Phi_{q}(\lambda, j)\right\|$ for $q \in\{1, \ldots, d\}$ (resp. $q \in\{d+1, \ldots, 2 d\})$ as $j$ tends to $+\infty$ (resp. $-\infty)$.

For the sake of simplicity, let us now drop the superscript \pm : from now on, $\mathrm{w}_{q}(\lambda)$ and $R_{q}(\lambda)$ will denote an eigenvalue and an associated eigenvector of $\mathbb{A}^{ \pm}(\lambda)$, and $a\left(\mathbf{w}_{q}\right)$ and $r\left(\mathbf{w}_{q}\right)$ will be the corresponding eigenvalue and eigenvectors of $F^{ \pm}$(see Tabs. 1 and 2).

There are two cases depending on the limit of $\mathrm{w}_{q}$ as $\lambda$ tends to 0 (see Tabs. 1 and 2), that is, whether the characteristic corresponding to the eigenvalue $a\left(\mathrm{w}_{q}\right)$ is entering or outgoing:

\section{- Case $\mathbf{j a}\left(\mathrm{w}_{\mathbf{q}}\right)<\mathbf{0}$ (entering characteristic)}

Recalling Tables 1 and 2, we see at once that, in this case, the index $q$ belongs to $\left\{1, \ldots, p^{+}\right\} \cup$ $\left\{d+p^{-}, \ldots, 2 d\right\}$. Thanks to Proposition 3.1, we know that the corresponding $\lambda \mapsto \nu_{q}(\lambda, l) \Phi_{q}(\lambda, j)$ is analytic for $q \in\left\{1, \ldots, p^{+}-\varpi-1\right\} \cup\left\{d+p^{-}+\varpi+1, \ldots, 2 d\right\}$ and meromorphic in $D(0, \sigma), \lambda=0$ 


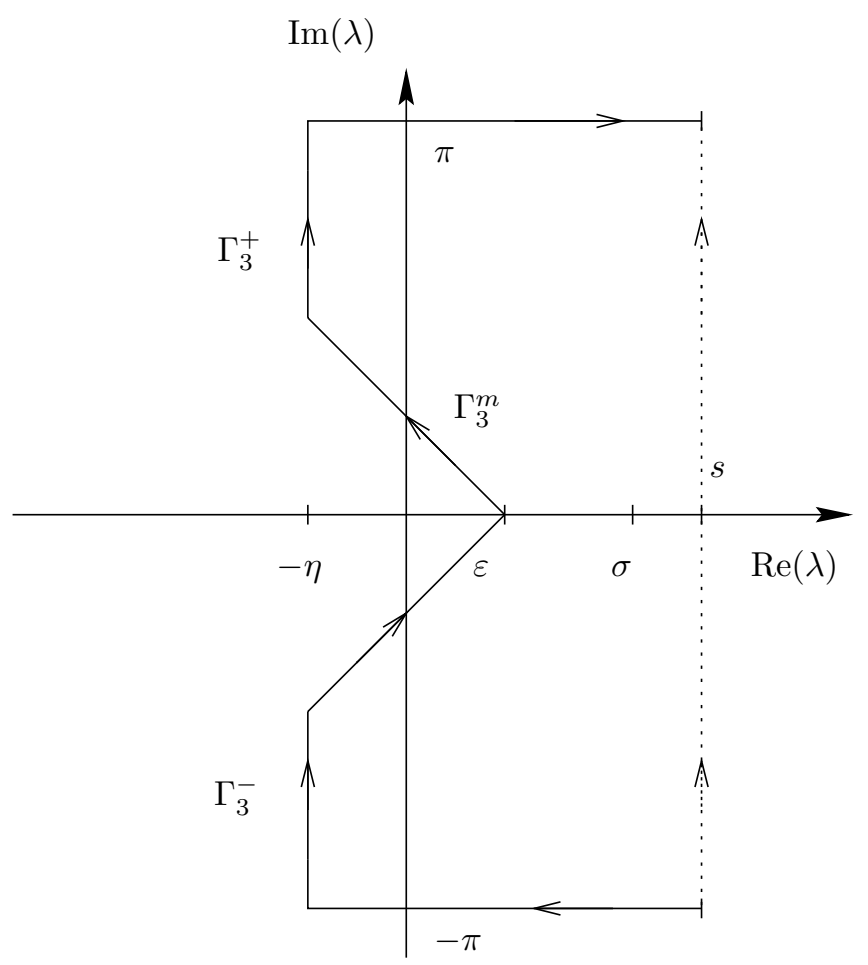

Figure 9. Path of integration $\Gamma_{3}$.

being its only pole for $q \in \Upsilon^{+} \cup \Upsilon^{-}$, so that

$$
\left\|\nu_{q}(\lambda, l) \Phi_{q}(\lambda, j)\right\| \leq \frac{C(\ell)}{|\lambda|}|\mathrm{w}(\lambda)|^{j}, \forall \lambda \in D(0, \sigma) \backslash\{0\} .
$$

Consequently, in the following, whenever the path of integration goes to the left-hand side of the point $\lambda=0$, a residue appears for $q \in \Upsilon^{+} \cup \Upsilon^{-}$.

The modulus of the associated eigenvalue $\left|w_{q}(0)\right|^{j}$ is strictly less than 1 , that is, choosing $\kappa>0$, if we take $\sigma$ to be small enough, we have, for $\lambda \in D(0, \sigma)$,

$$
j \ln \left(\left|\mathrm{w}_{q}(\lambda)\right|\right) \leq-\kappa|j| .
$$

Let us consider at first the case $n \leq|j / a|$ : this artificial choice of bound is explained by the bounds appearing in the case of $j a>0$. Let $\varepsilon$ be such that that $0<\varepsilon<\min (\kappa|a|, \sigma) / 2$, so that our path of integration

$$
\Gamma_{3}:=\Gamma_{3}^{-} \cup \Gamma_{3}^{m} \cup \Gamma_{3}^{+},
$$

with $\Gamma_{3}^{m}:=\{|\operatorname{Im}(\lambda)|=\varepsilon-\operatorname{Re}(\lambda),-\eta \leq \operatorname{Re}(\lambda) \leq \varepsilon\}$, is such that $\Gamma_{3}^{m}$ intersects $\{\operatorname{Re}(\lambda)=-\eta$ inside $D(0, \sigma)$ (see Fig. 9). Using once again the notations of (80), we obtain

$$
\left|I_{3}^{-}\right|+\left|I_{3}^{+}\right| \leq C(\ell) \mathrm{e}^{-\eta n} .
$$


Noting that $|\lambda| \geq \varepsilon / \sqrt{2}$ for $\lambda \in \Gamma_{3}$, we obtain

$$
|\mathrm{G}(n, l, j)| \leq C(\ell) \mathrm{e}^{\varepsilon n-\kappa|j|} \leq C(\ell) \mathrm{e}^{-\kappa|a| n / 2} .
$$

No residue appears since $\lambda=0$ remains on the left-hand side of $\Gamma_{3}$. We find the right estimate using Remark 4.2.

Let us consider now the case $n>|j / a|$. We integrate along $\Gamma_{0}$ (see Fig. 8). Noting that $\left\|\nu_{q}(\lambda, l) \Phi_{q}(\lambda, j)\right\| \leq C(\ell) \mathrm{e}^{-\kappa|j|} \leq C(\ell)$, it is the same computation as in the case of $|l-j| \leq \ell$ and large $n$ : a residue appears in the terms corresponding to $q \in \Upsilon^{+} \cup \Upsilon^{-}$. We conclude again by using Remark 4.2.

- Case $\mathbf{j a}\left(\mathrm{w}_{\mathbf{q}}\right)>\mathbf{0}$ (outgoing characteristic)

Referring to Tables 1 and 2 , we know that $\mathrm{w}_{q}(\lambda)$ tends to 1 as $\lambda$ tends to 0 , so that the index $q$ in the integral (82) belongs to $\left\{p^{+}+1, \ldots, d\right\} \cup\left\{d+1, \ldots, d+p^{-}-1\right\}$ : Proposition 3.1 implies that the corresponding $\lambda \mapsto \nu_{q}(\lambda, l)$ is holomorphic in $D(0, \sigma)$, so that

$$
\left\|\nu_{q}(\lambda, l)\right\| \leq C(\ell)
$$

Let us now drop the subscript $q$ and note $a:=a\left(\mathrm{w}_{q}\right)$ and $r:=r\left(\mathrm{w}_{q}\right)$. We derive an expansion of $\ln (|\mathrm{w}(\lambda)|)=\ln (\mathrm{w}(\lambda) \overline{\mathrm{w}(\lambda)}) / 2$ from the expansion $(38)$ :

$$
\ln (|\mathrm{w}(\lambda)|)=-\frac{\operatorname{Re}(\lambda)}{a}+\frac{\alpha}{2 a}\left(\operatorname{Re}(\lambda)^{2}-\operatorname{Im}(\lambda)^{2}\right)+o\left(|\lambda|^{2}\right)
$$

where

$$
\alpha:=\frac{2 D}{a^{2}}-1
$$

Note that $\alpha>0$ because $a^{2}<|a|<2 D<1$ (H5). Define

$$
\xi:=\frac{\frac{j}{a}-n}{\alpha \frac{j}{a}} .
$$

Following the method of [27], let us change the initial path of integration $\Gamma$ to $\Gamma_{4}:=\Gamma_{4}^{-} \cup \mathcal{H}_{\lambda_{0}} \cup \Gamma_{4}^{+}$, $\mathcal{H}_{\lambda_{0}}$ being the portion contained in $\operatorname{Re}(\lambda) \geq-\eta$ of the hyperbola

$$
-\operatorname{Re}(\lambda)+\frac{\alpha}{2}\left(\operatorname{Re}(\lambda)^{2}-\operatorname{Im}(\lambda)^{2}\right)=-\lambda_{0}+\frac{\alpha}{2} \lambda_{0}^{2},
$$

that intersects the real axis at

$$
\begin{aligned}
\lambda_{0} & :=\xi, & & \text { if }|\xi| \leq \epsilon, \\
& :=\epsilon, & & \text { if } \xi>\epsilon, \\
& :=-\epsilon, & & \text { if } \quad-\epsilon>\xi
\end{aligned}
$$

where $\epsilon$ is chosen small enough to ensure that $\mathcal{H}_{\epsilon}$ intersects $\Gamma_{0}$ inside $D(0, \sigma)$ (see Figs. 10 and 11). These choices are carefully explained in [27].

We have the following expansion for $\lambda \in \mathcal{H}_{\lambda_{0}}$

$$
\operatorname{Re}(\lambda)=\lambda_{0}-\frac{\alpha}{2} \operatorname{Im}(\lambda)^{2}+O\left(|\operatorname{Im}(\lambda)|^{3}\right)
$$




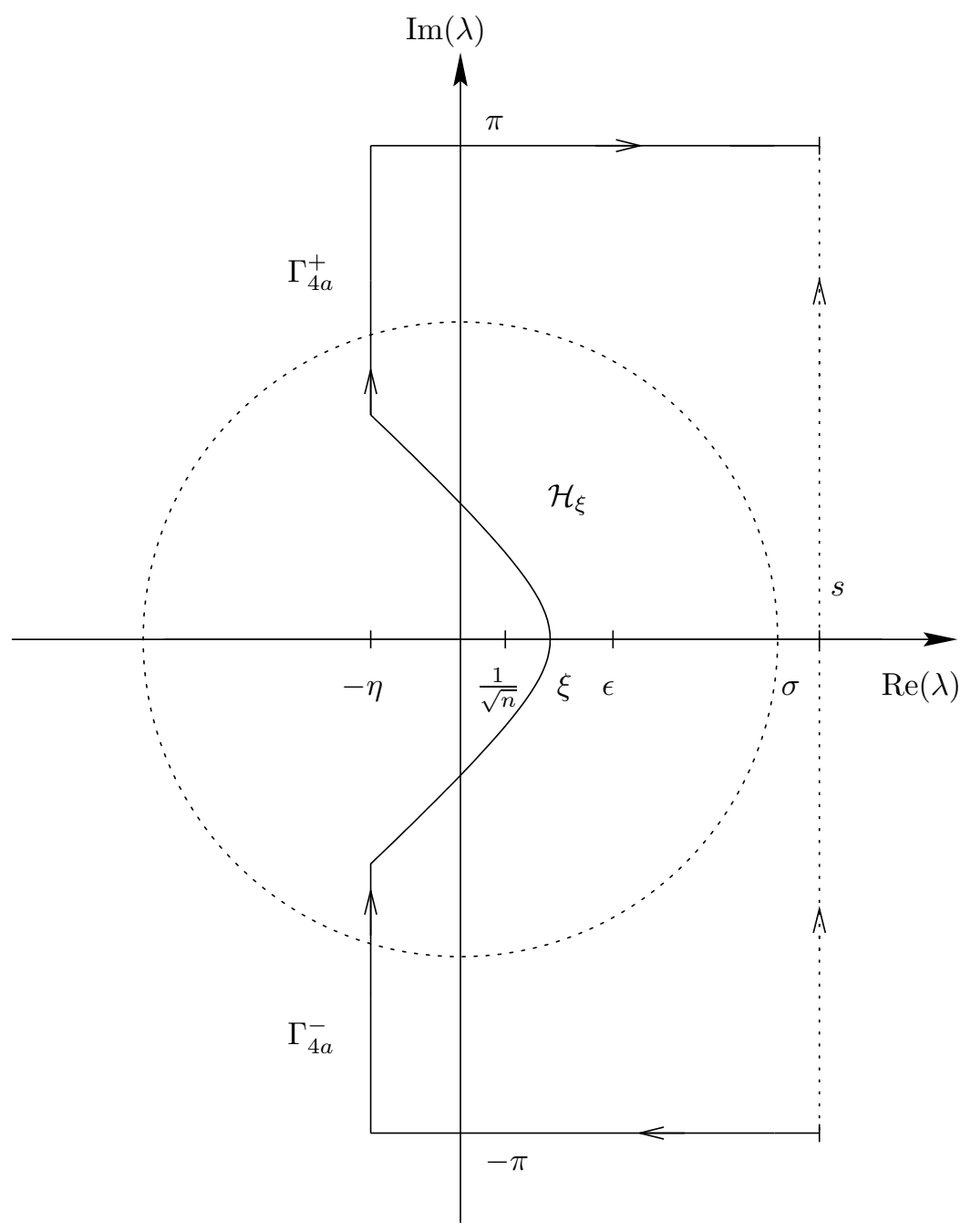

Figure 10. Path of integration $\Gamma_{4 a}$.

that leads to

$$
C_{1}\left(\left|\lambda_{0}\right|+|\operatorname{Im}(\lambda)|\right) \leq|\lambda| \leq C_{2}\left(\left|\lambda_{0}\right|+|\operatorname{Im}(\lambda)|\right)
$$

where $C_{1}$ and $C_{2}$ are some positive constants. Using (87), we have for $m \in \mathbb{N} \backslash\{0\}$

$$
|\lambda|^{m} \leq C\left(\left|\lambda_{0}\right|^{m}+|\operatorname{Im}(\lambda)|^{m}\right)
$$

Recall that, if $\xi$ is negative, that is, if $j / a<n$, the path $\mathcal{H}_{\lambda_{0}}$ lies in the left half-plane. But here, we are dealing with $\lambda \mapsto \nu(\lambda, l) \Phi(\lambda, j)$ that are holomorphic in $D(0, \sigma)$, so that no residue appears. Using the notations of $(80)$ for $\nu(\lambda, l) \Phi(\lambda, j)$ instead of $\mathrm{G}_{\lambda}(l, j)$, we obtain at once

$$
\left|I_{4}^{-}\right|+\left|I_{4}^{+}\right| \leq C(\ell) \mathrm{e}^{-\eta n}
$$




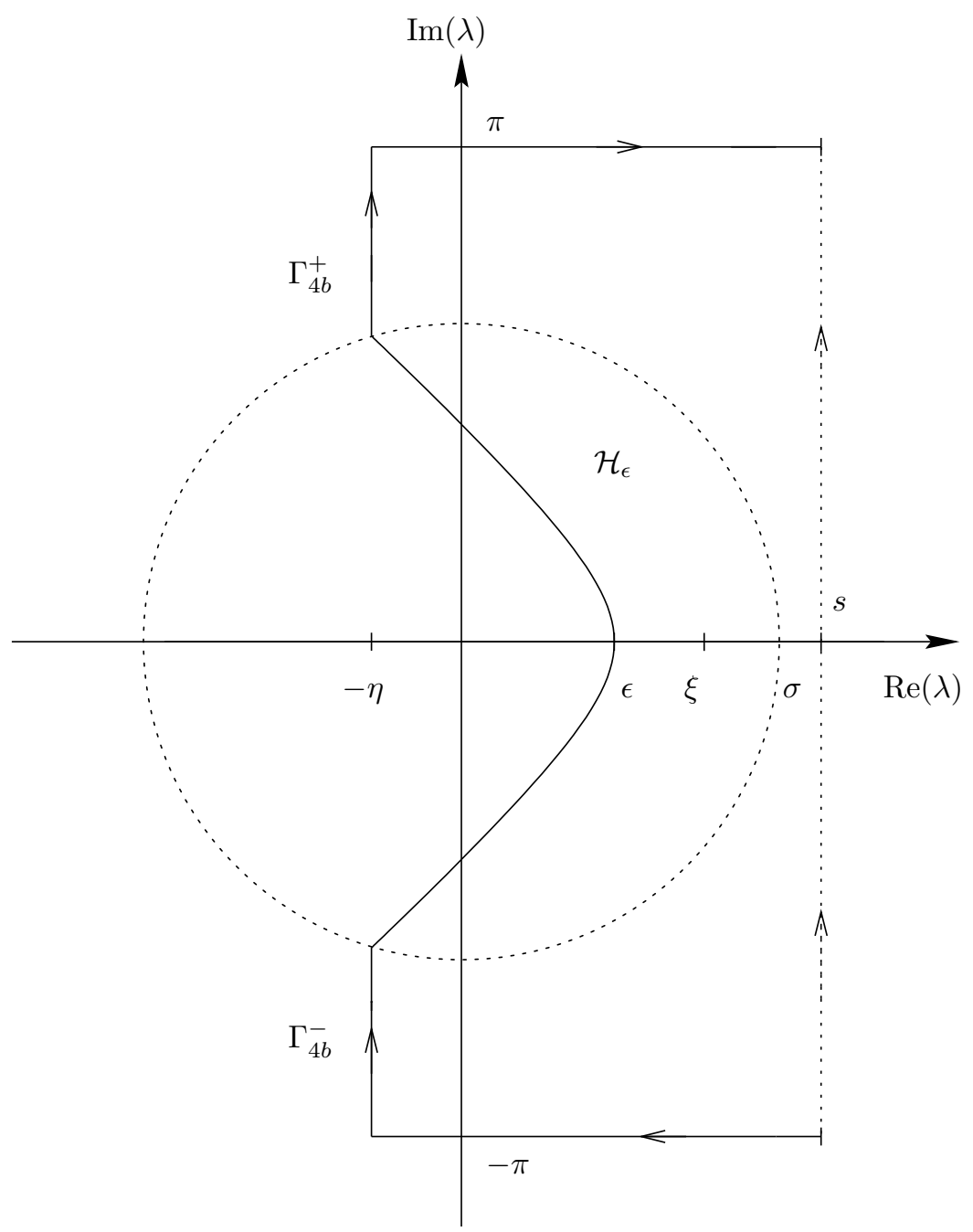

Figure 11. Path of integration $\Gamma_{4 b}$.

Let us now focus on the integration along $\mathcal{H}_{\lambda_{0}}$. Remembering the behaviors (41) of the solutions of $(35)$ in $D(0, \sigma)$, we can write

$$
\begin{aligned}
\left|I_{4}^{m}\right| & \leq C(\ell) \int_{\mathcal{H}_{\lambda_{0}}} \mathrm{e}^{\operatorname{Re}(\lambda) n}|\mathrm{w}(\lambda)|^{j}|R(\lambda)||\mathrm{d} \lambda|+O\left(\int_{\mathcal{H}_{\lambda_{0}}} \mathrm{e}^{\operatorname{Re}(\lambda) n}|\mathrm{w}(\lambda)|^{j} \omega^{-|j|}|\mathrm{d} \lambda|\right) \\
& \leq C(\ell) \int_{\mathcal{H}_{\lambda_{0}}} \mathrm{e}^{\varphi(n, j, \lambda)}\left|\left(\begin{array}{c}
r \\
-\frac{\lambda}{a} r
\end{array}\right)+O\left(\begin{array}{c}
|\lambda| \\
|\lambda|^{2}
\end{array}\right)\right||\mathrm{d} \lambda|+\omega^{-|j|} O\left(\int_{\mathcal{H}_{\lambda_{0}}} \mathrm{e}^{\varphi(n, j, \lambda)}|\mathrm{d} \lambda|\right) \\
& =J_{1}+J_{2}
\end{aligned}
$$

where

$$
\varphi(n, j, \lambda):=\operatorname{Re}(\lambda) n+j \ln (|\mathrm{w}(\lambda)|)
$$


Note that $|\mathrm{d} \lambda| \leq C_{4}$, for some positive $C_{4}$ and that, since $|\xi| \leq \epsilon$, we have

$$
(1-\alpha \sigma) \frac{j}{a} \leq n \leq(1+\alpha \sigma) \frac{j}{a} .
$$

Case $|\boldsymbol{\xi}| \leq \boldsymbol{\epsilon}$ (see Fig. 10)

Using (86), we get

$$
\begin{aligned}
\varphi(n, j, \lambda) & =n(\operatorname{Re}(\lambda)-\xi)+n \xi-\frac{j}{a}\left(\xi-\frac{\alpha}{2} \xi^{2}\right)+O\left(|\lambda|^{3}\right) \\
& =-\frac{\alpha}{2} n \operatorname{Im}(\lambda)^{2}-\frac{\left(\frac{j}{a}-n\right)^{2}}{2 \alpha \frac{j}{a}}+\frac{j}{a} O\left(|\lambda|^{3}\right) .
\end{aligned}
$$

Thanks to (88), we obtain

$$
\begin{aligned}
\varphi(n, j, \lambda) & \leq \frac{j}{a}\left(-\frac{\alpha}{2} \xi^{2}+O\left(|\xi|^{3}\right)\right)-\frac{j}{a}\left(\frac{\alpha}{2} \operatorname{Im}(\lambda)^{2}+O\left(\left|\operatorname{Im}(\lambda)^{3}\right|\right)\right) \\
& \leq-\frac{j}{a} \frac{\xi^{2}}{M}-\frac{j}{a} \frac{\operatorname{Im}(\lambda)^{2}}{M^{\prime}}
\end{aligned}
$$

for some positive $M, M^{\prime}$. Let us now denote by

$$
\bar{\xi}:=\frac{\frac{j}{a}-n}{n}
$$

the variable we expect to appear by comparison with the continuous case that is treated in [27] for the shock wave setting and in [12] for the pure boundary layer one. Choosing $\sigma$ to be small enough, (90) implies that there exists $c>0$ such that

$$
|\bar{\xi}| \leq c \epsilon
$$

Since $|\xi| \leq \epsilon$, we have

$$
\begin{aligned}
\frac{j}{a} \frac{\xi^{2}}{M} & =\frac{\left(\frac{j}{a}-n\right)^{2}}{M \alpha^{2} \frac{j}{a}}=\frac{\left(\frac{j}{a}-n\right)^{2}}{M \alpha^{2} n}\left(1+O\left(\frac{\frac{j}{a}-n}{n}\right)\right) \\
& =n \frac{\bar{\xi}^{2}}{M \alpha^{2}}+n O\left(\bar{\xi}^{3}\right) \\
& \leq n \frac{\bar{\xi}^{2}}{M^{\prime \prime}}
\end{aligned}
$$

where $M^{\prime \prime}$ is a positive constant.

Let us deal at first with the second term $J_{2}$ of (89).

We get at once

$$
J_{2} \leq C(\ell) \omega^{-|j|} \int_{\mathcal{H}_{\lambda_{0}}} \mathrm{e}^{\varphi(n, l, j)}|\mathrm{d} \lambda| .
$$


The bounds given in (90) imply that there exists a positive $\gamma$ such that

$$
\omega^{-|j|} \leq C \mathrm{e}^{-\gamma n}
$$

so that (91) and (93) imply

$$
J_{2} \leq C(\ell) \mathrm{e}^{-\gamma n} \mathrm{e}^{-n \bar{\xi}^{2} / M^{\prime \prime}}
$$

Remark 4.3. Note that (94) is satisfied in the two cases on $\xi$ we consider.

Let us now deal with the first term $J_{1}$ of (89), we integrate the term of order 0 with respect to $\lambda$.

$$
\begin{aligned}
\int_{\mathcal{H}_{\lambda_{0}}} \mathrm{e}^{\varphi(n, j, \lambda)}|\mathrm{d} \lambda| & \leq \mathrm{e}^{-n \bar{\xi}^{2} / M^{\prime \prime}} \int_{\mathcal{H}_{\lambda_{0}}} \exp \left(-\frac{j}{a} \frac{y^{2}}{M^{\prime}}\right) \mathrm{d} y \\
& \leq \mathrm{e}^{-n \bar{\xi}^{2} / M^{\prime \prime}} \sqrt{\frac{a M^{\prime}}{j}} \int_{-\infty}^{+\infty} \mathrm{e}^{-y^{2}} \mathrm{~d} y
\end{aligned}
$$

Using (90), we have

$$
\sqrt{n} \sqrt{\frac{a}{j}} \leq C_{4}
$$

where $C_{4}$ is a positive constant that does not depend on $n$ nor on $j$. In conclusion, we get

$$
\int_{\mathcal{H}_{\lambda_{0}}} \mathrm{e}^{\varphi(n, j, \lambda)}|\mathrm{d} \lambda|=\frac{1}{\sqrt{n}} O\left(\mathrm{e}^{-n \bar{\xi}^{2} / M^{\prime \prime}}\right) .
$$

Using (88) for $m \in\{1,2\}$, (91) and (93), we have

$$
\begin{aligned}
\int_{\mathcal{H}_{\lambda_{0}}}|\lambda|^{m} \mathrm{e}^{\varphi(n, j, \lambda)}|\mathrm{d} \lambda| & \leq \mathrm{e}^{-n \bar{\xi}^{2} / M^{\prime \prime}} \int_{-\infty}^{+\infty} C_{5}\left(|\bar{\xi}|^{m}+|y|^{m}\right) \mathrm{e}^{-j y^{2} /\left(a M^{\prime}\right)} \mathrm{d} y \\
& \leq C_{6}\left(|\bar{\xi}|^{m} \mathrm{e}^{-n \bar{\xi}^{2} / M^{\prime \prime}}+\mathrm{e}^{-n \bar{\xi}^{2} / M^{\prime \prime}} \int_{-\infty}^{+\infty}|y|^{m} \mathrm{e}^{-j y^{2} /\left(a M^{\prime}\right)} \mathrm{d} y\right)
\end{aligned}
$$

where $C_{5}$ and $C_{6}$ are positive constants.

Using the well-known inequality

$$
|X| \mathrm{e}^{-X^{2}} \leq \mathrm{e}^{-X^{2} / 2}
$$

we finally get

$$
\int_{\mathcal{H}_{\lambda_{0}}}|\lambda|^{m} \mathrm{e}^{\varphi(n, j, \lambda)}|\mathrm{d} \lambda|=\frac{1}{n} O\left(\mathrm{e}^{-n \bar{\xi}^{2} / M^{\prime \prime}}\right) .
$$

In the next case, we only develop the estimates of $\varphi(n, l, j)$, since the remaining computations are exactly the same as in the case we just considered.

Case $|\xi|>\epsilon$

We only treat here the case $\xi>\epsilon$ (see Fig. 11) because the case $\xi<-\epsilon$ is completely similar. 
Using the expansion (86) and (88), we obtain

$$
\begin{aligned}
\varphi(n, j, \lambda) & =n(\operatorname{Re}(\lambda)-\epsilon)+\left(n-\frac{j}{a}\right) \epsilon+\frac{j}{a} \frac{\alpha}{2} \epsilon^{2}+O\left(|\lambda|^{3}\right) \\
& =-n \frac{\alpha}{2} \operatorname{Im}(\lambda)^{2}+\frac{j}{a} O\left(|\operatorname{Im}(\lambda)|^{3}\right)-\frac{j}{a}\left(\frac{\alpha}{2} \epsilon^{2}+O\left(\epsilon^{3}\right)\right) \\
& \leq-n \frac{\operatorname{Im}(\lambda)^{2}}{M^{\prime}}-\frac{j}{a} \frac{\epsilon^{2}}{M} \\
& \leq-n \frac{\operatorname{Im}(\lambda)^{2}}{M^{\prime}}-n \frac{\bar{\xi}^{2}}{M^{\prime \prime}} .
\end{aligned}
$$

We get the claimed estimates through the same computations as in the previous case.

\section{Numerical SIMULATiONS}

We show here some numerical simulations that we obtained by considering a Lax 3 -shock for the $3 \times 3$ system of gas dynamics

$$
\begin{aligned}
\rho_{t}+(\rho v)_{x} & =0 \\
(\rho v)_{t}+\left(\rho v^{2}+(\gamma-1) \rho e\right)_{x} & =0 \\
\left(\frac{1}{2} \rho v^{2}+\rho e\right)_{t}+\left(v\left(\frac{1}{2} \rho v^{2}+\gamma \rho e\right)\right)_{x} & =0
\end{aligned}
$$

where $\rho$ is the density of the gas, $v$ its velocity and $e$ its internal energy. We take here the law of pressure of the perfect gases:

$$
P(\rho, e)=(\gamma-1) \rho e
$$

The adiabatic constant $\gamma$ is larger than 1 ; physically, $\gamma$ is equal to $5 / 3$ for monatomic gases and $7 / 5$ for diatomic gases. Taking the same notations as in [3], we have

$$
u=\left(\begin{array}{c}
\rho \\
\rho v \\
\frac{1}{2} \rho v^{2}+\rho e
\end{array}\right), f(u)=\left(\begin{array}{c}
\rho v \\
\rho v^{2}+(\gamma-1) \rho e \\
v\left(\frac{1}{2} \rho v^{2}+\gamma \rho e\right)
\end{array}\right)
$$

Let $u^{-}=\left(\rho^{-}, \rho^{-} v^{-}, \frac{1}{2} \rho^{-}\left(v^{-}\right)^{2}+\rho^{-} \mathrm{e}^{-}\right)^{T}$ and $u^{+}=\left(\rho^{+}, \rho^{+} v^{+}, \frac{1}{2} \rho^{+}\left(v^{+}\right)^{2}+\rho^{+} \mathrm{e}^{+}\right)^{T}$ two states of $\mathbb{R}^{3}$ satisfying the Rankine-Hugoniot condition (H1) with null speed

$$
\begin{aligned}
\rho^{+} v^{+}-\rho^{-} v^{-} & =0, \\
\rho^{+}\left(v^{+}\right)^{2}+(\gamma-1) \rho^{+} \mathrm{e}^{+}-\rho^{-}\left(v^{-}\right)^{2}-(\gamma-1) \rho^{-} \mathrm{e}^{-} & =0, \\
v^{+}\left(\frac{1}{2} \rho^{+}\left(v^{+}\right)^{2}+\gamma \rho^{+} \mathrm{e}^{+}\right)-v^{-}\left(\frac{1}{2} \rho^{-}\left(v^{-}\right)^{2}+\gamma \rho^{-} \mathrm{e}^{-}\right) & =0 .
\end{aligned}
$$


In order to be able to apply the modified Lax-Friedrichs scheme to the system $u_{t}+f(u)_{x}=0$, we change to the conservative variables

$$
R=\rho, \quad V=\rho v, \quad E=\frac{1}{2} \rho v^{2}+\rho e,
$$

and

$$
U=\left(\begin{array}{l}
R \\
V \\
E
\end{array}\right), F(U)=f(u)=\left(\begin{array}{c}
V \\
\frac{3-\gamma}{2} \frac{V^{2}}{R}+(\gamma-1) E \\
\frac{V}{R}\left(\gamma E-\frac{\gamma-1}{2} \frac{V^{2}}{R}\right)
\end{array}\right)
$$

We get at once

$$
\mathrm{d} F(U)=\left(\begin{array}{ccc}
0 & 1 & 0 \\
\frac{\gamma-3}{2} \frac{V^{2}}{R^{2}} & (3-\gamma) \frac{V}{R} & \gamma-1 \\
\frac{V}{R}\left((\gamma-1) \frac{V^{2}}{R^{2}}-\gamma \frac{E}{R}\right) & \gamma \frac{E}{R}-3 \frac{(\gamma-1)}{2} \frac{V^{2}}{R^{2}} & \gamma \frac{V}{R}
\end{array}\right)
$$

The eigenvalues of $\mathrm{d} F(U)$ are

$$
a_{1}(U)=\frac{V}{R}-c(U), a_{2}(U)=\frac{V}{R}, a_{3}(U)=\frac{V}{R}+c(U),
$$

where $c(U)$ is the sound speed and is given by

$$
c(U)=\sqrt{\gamma(\gamma-1)\left(\frac{E}{R}-\frac{V^{2}}{2 R^{2}}\right)} .
$$

We choose as associated eigenvectors

$$
r_{1}(U)=\left(\begin{array}{c}
R \\
V-R c(U) \\
\frac{(1-\gamma)}{2} \frac{V^{2}}{R}+\gamma E-V c(U)
\end{array}\right), r_{2}(U)=\left(\begin{array}{c}
R \\
V \\
\frac{V^{2}}{2 R}
\end{array}\right), r_{3}(U)=\left(\begin{array}{c}
R \\
V+R c(U) \\
\frac{(1-\gamma)}{2} \frac{V^{2}}{R}+\gamma E+V c(U)
\end{array}\right)
$$

The stationary discontinuity $\left(U^{-}, U^{+}\right)$is a Lax 3 -shock, that is (H4) is satisfied with $p^{+}=p^{-}=3$, so that

$$
\begin{gathered}
V^{+}+R^{+} c^{+}<0<V^{-}+R^{-} c^{-}, \\
V^{-}<0
\end{gathered}
$$

where $c^{ \pm}:=c\left(U^{ \pm}\right)$. Consequently, plugging (98) and (99) in (95)-(97), we can completely define the end states $U^{ \pm}$by three parameters $\left(R^{-}, r:=R^{-} / R^{+}, V^{-}\right)$in the following way

$$
\begin{aligned}
R^{-}, & R^{+}=r^{-1} R^{-}, \\
V^{-}, & V^{+}=V^{-}, \\
E^{-}=\frac{\gamma^{2}+\gamma(r-2)+r+1}{2 \gamma(\gamma-1)}\left(\frac{\left(V^{-}\right)^{2}}{R^{-}}\right), & E^{+}=\frac{r \gamma(r-1)+r+1+\gamma(\gamma-1)}{2 \gamma(\gamma-1)}\left(\frac{\left(V^{-}\right)^{2}}{R^{-}}\right),
\end{aligned}
$$



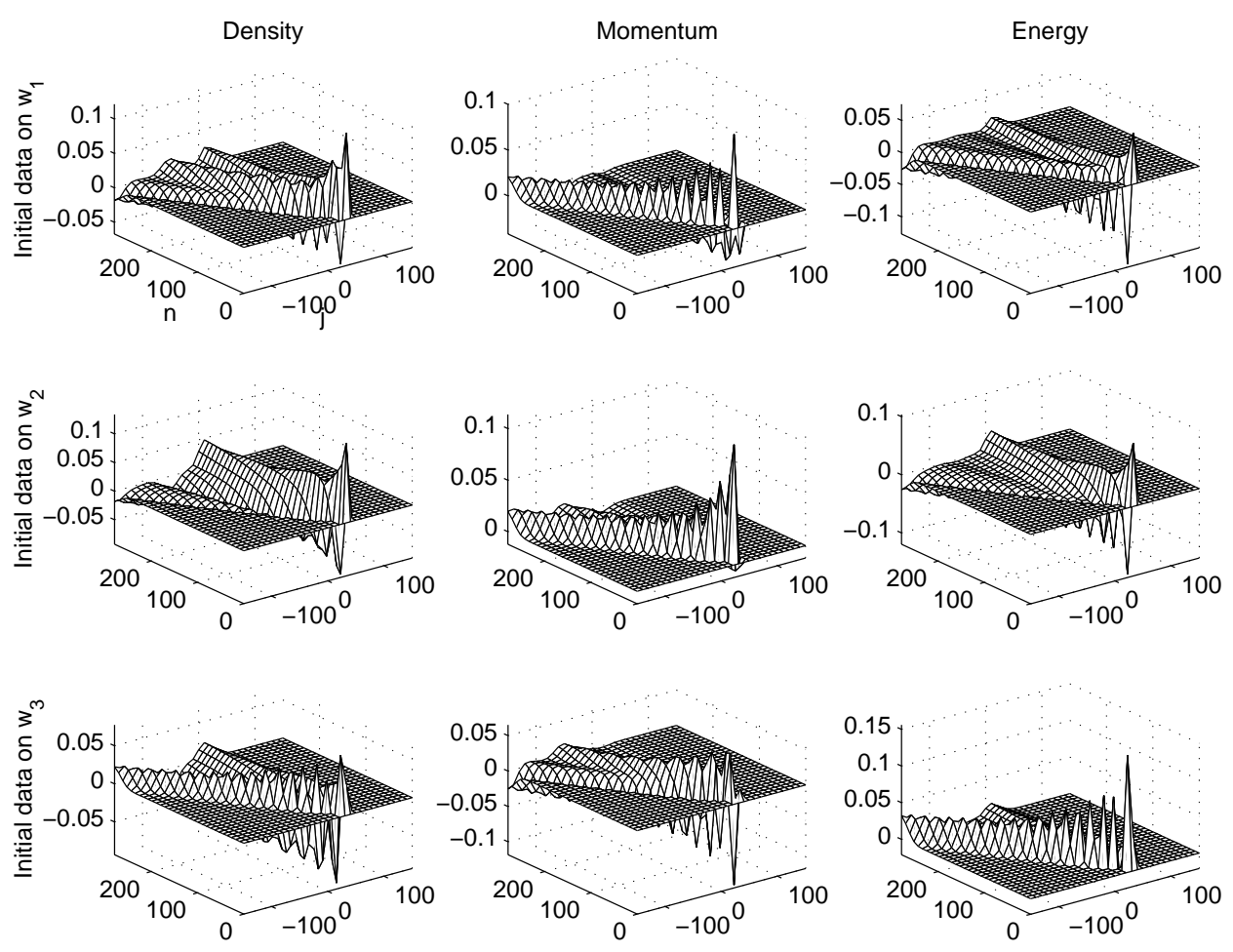

Figure 12. $\mathrm{G}(n, 39, j)$ displayed on the canonical basis $\left(w_{1}, w_{2}, w_{3}\right)$.

where

$$
1<r<\frac{\gamma+1}{\gamma-1}
$$

(see [3] for details).

Referring to Table 1 and to (41), we see at once that $\Phi_{6}^{\mathrm{I}}=\Psi$, and that $\Psi(j)=\left(\zeta_{3}^{-}\right)^{j}\left(r_{3}^{-}+O\left(\omega^{-|j|}\right)\right)$ as $j$ tends to $-\infty$.

The algorithm is the following:

1. We choose the size $N$ of the mesh.

2. We set the values of $R^{-}, r$ and $V^{-}$and compute $U^{ \pm}$.

3. Having computed the eigenvalues of $\mathrm{d} F\left(U^{ \pm}\right)$, we set $D=1.1 * \max \left(\left|a_{i}^{ \pm}\right|, i \in\{1,2,3\}\right)$ and $\sigma=0.9 /(2 * D)$ so that (H5) is satisfied.

4. We compute numerically a profile by iterating the Lax-Friedrichs scheme in conservative coordinates on the step sequence $U_{j}=U^{-}$if $j \leq 0$ and $U_{j}=U^{-}$if $j \geq 1$ : the convergence is quite fast [13].

5. We choose the location of the initial data, $l \in\{-N / 2, \ldots, N / 2\}$ and its direction $\mathbf{V}$.

6. We solve numerically (9) with $\tilde{v}=0$ and $v_{j}^{0}=\delta_{j l} \mathbf{V}$.

Let us consider the cases $l \geq 0$ and $l \leq 0$ :

Case $l \geq 0$ in accordance with Theorem 1.1, in Figure 12, three waves propagate towards the shock with speeds $a_{1}^{+}, a_{2}^{+}, a_{3}^{+}$since the three of them are negative. When the fastest one reaches the shock, i.e. the one corresponding to $a_{1}^{+}$, a residue appears (see Fig. 13), and outgoing waves are emitted on the left side with speeds $a_{1}^{-}$and $a_{2}^{-}$along the eigenvectors $r_{1}^{-}$and $r_{2}^{-}$. We cannot see clearly in Figure 12 

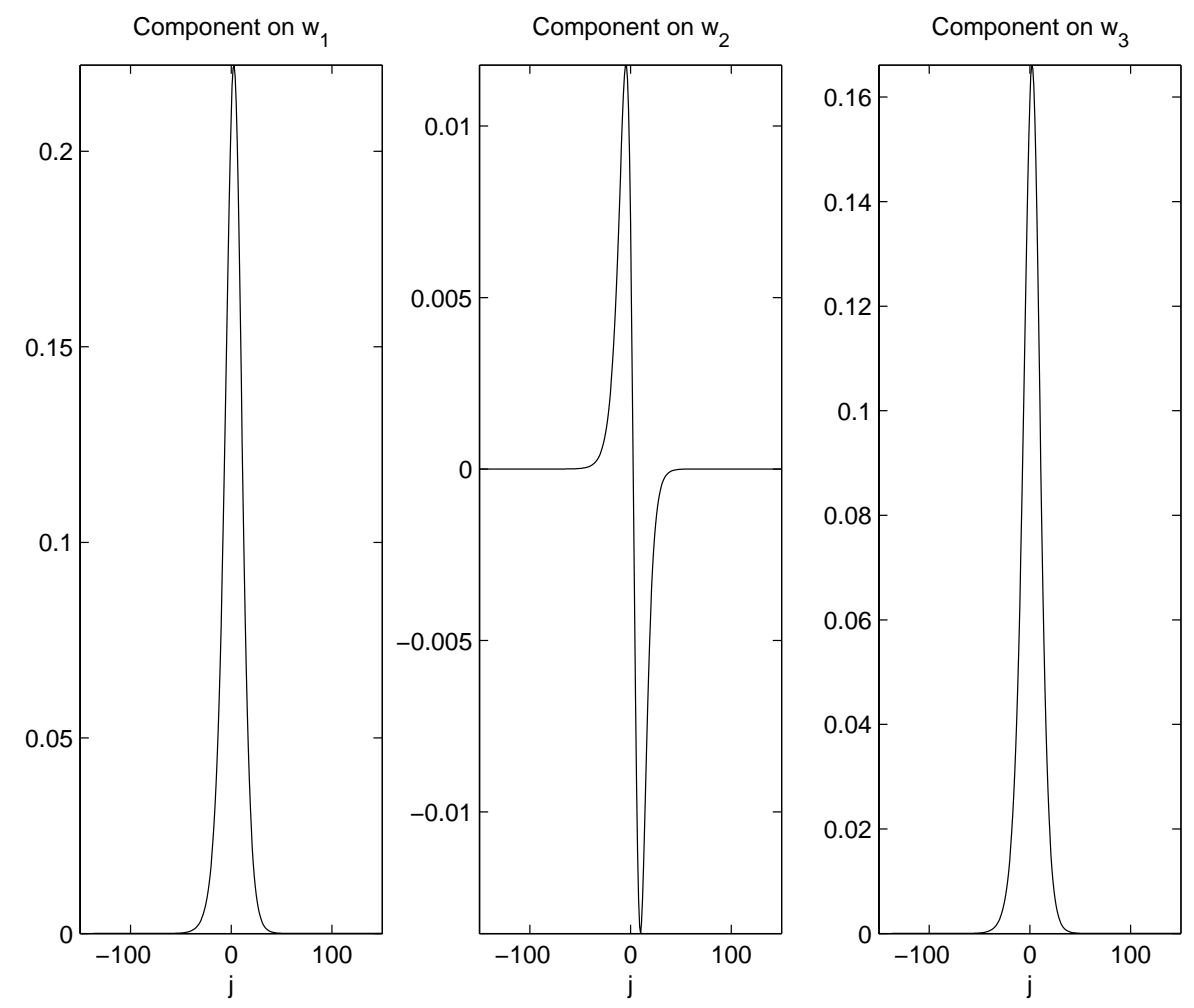

FIGURE 13. Eigenspace associated to 1 in the canonical basis.

the waves that are emitted by the waves that are carried by $r_{1}^{+}$and $r_{2}^{+}$because they are damped by the numerical viscosity and, besides, the scale of the residue is large and the waves are all the more damped. No wave outgoes on the right since the eigenvalues $a_{1}^{+}, a_{2}^{+}, a_{3}^{+}$are all negative.

Case $l \leq 0$ Since all the eigenvalues of $\mathrm{d} f\left(u^{+}\right)$are negative, no wave propagates to the right: all the waves are in the left side of the mesh. Thus, since all the waves propagate along $r_{1}^{-}, r_{2}^{-}$and $r_{3}^{-}$, we chose the three of them as a basis for the computations we show in Figure 14. We see that a single wave propagates along the entering characteristic with speed $a_{3}^{-}>0$ until it reaches the shock; then, a stationary residue appears (see Fig. 15 for the projection on each vector of the basis $\left(r_{1}^{-}, r_{2}^{-}, r_{3}^{-}\right)$of the eigenspace associated with 1 ), along with two waves propagating to the left with speeds $a_{1}^{-}<0$ and $a_{2}^{-}<0$.

In order to compare the numerical results to our expectations a time step at a time, we resume our study with

6. We compute the residue up to a multiplicative constant by iterating the linearized scheme (8) from $-3 N / 4$ to $N / 2$ on the initial data $\Psi(-3 N / 4)=\left(\zeta_{3}^{-}\right)^{-3 N / 4} r_{3}^{-}$.

7. We compute the Gaussians through the formula given in Theorem 1.1.

Movies displaying the evolution of the Green's function with respect to time are available at http://www . umpa.ens-lyon.fr/ pgodillo.

Acknowledgements. I would like to thank Emmanuel Grenier for suggesting this problem to me and answering my numerous questions, Frédéric Rousset for many fruitful discussions and Kevin Zumbrun for his advice and encouragements. 

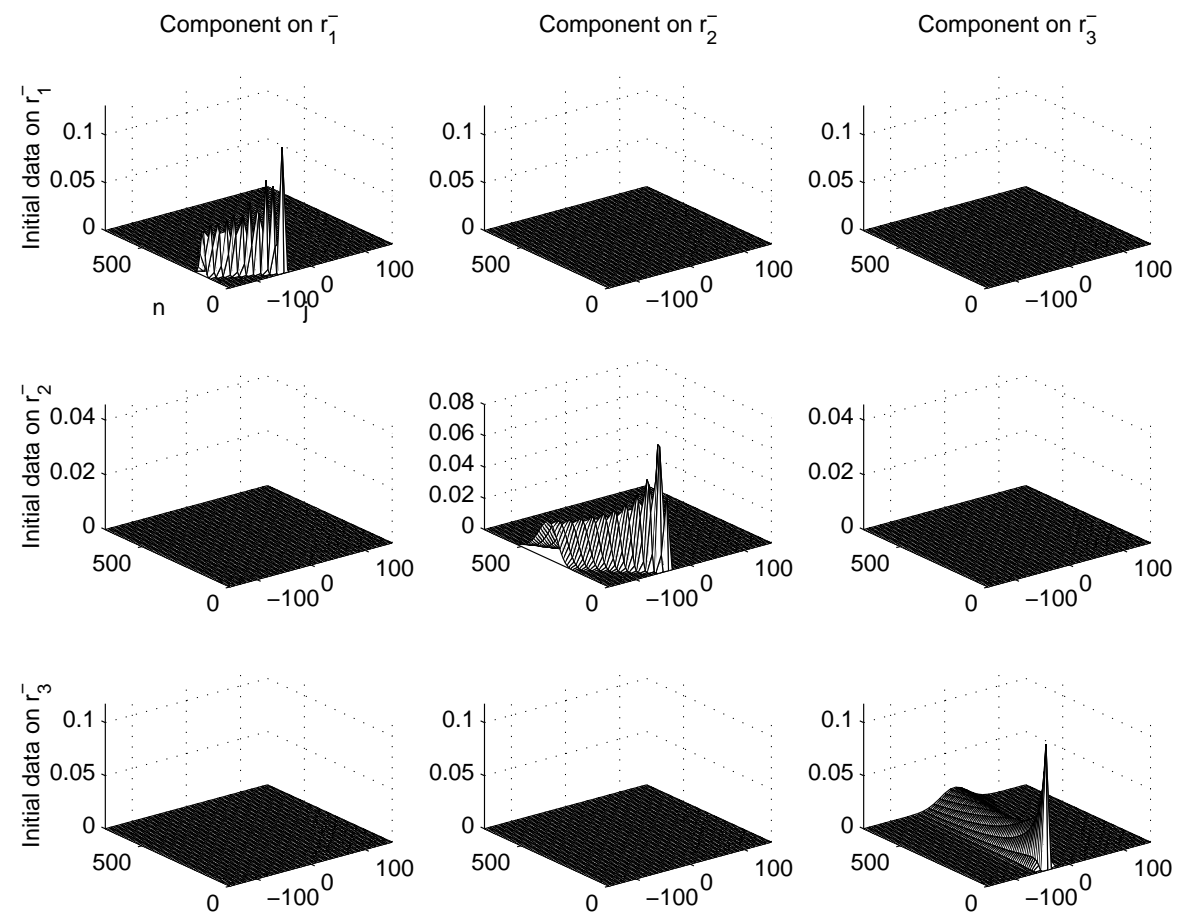

Figure 14. $\mathrm{G}(n,-41, j)$ displayed on the basis $\left(r_{1}^{-}, r_{2}^{-}, r_{3}^{-}\right)$.
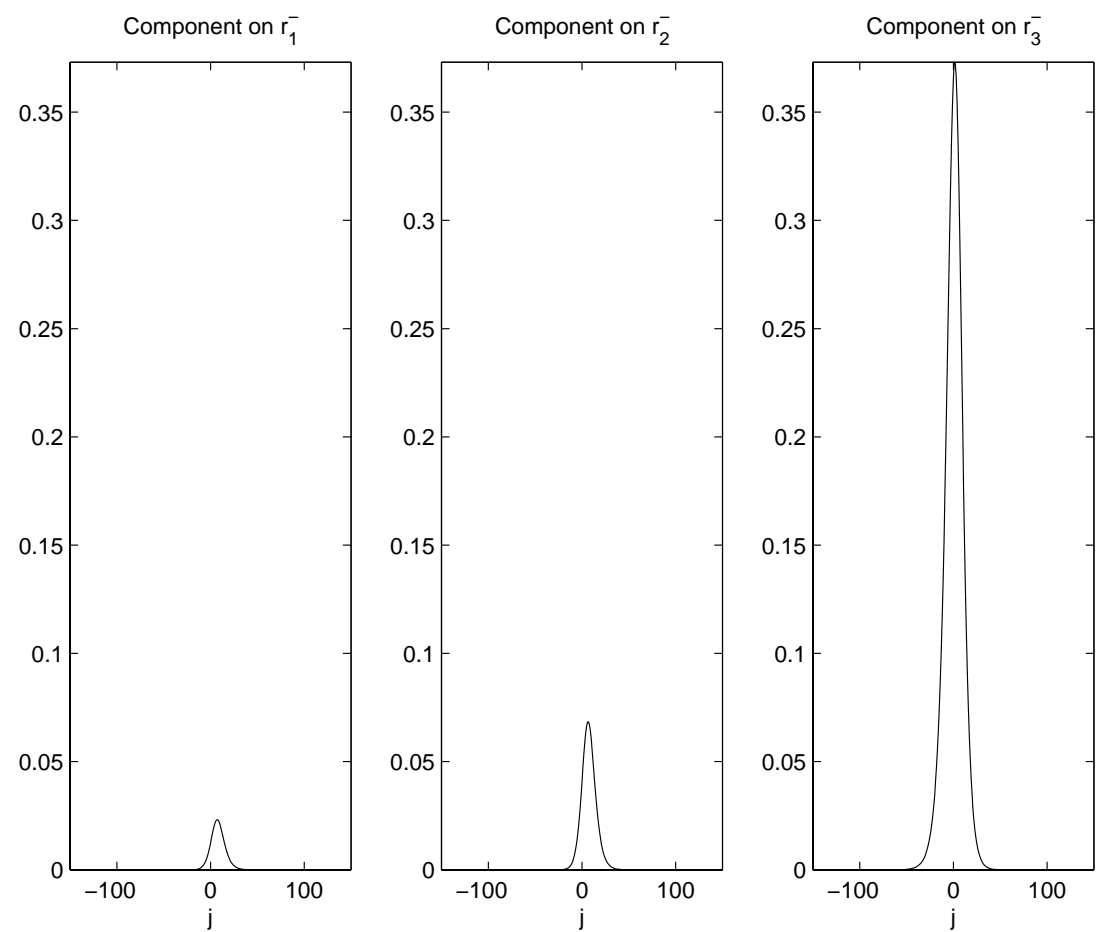

FiguRE 15 . Eigenspace associated to 1 in the basis $\left(r_{1}^{-}, r_{2}^{-}, r_{3}^{-}\right)$. 


\section{REFERENCES}

[1] S. Benzoni-Gavage, Stability of semi-discrete shock profiles by means of an Evans function in infinite dimensions. J. Dynam. Differential Equations 14 (2002) 613-674.

[2] S. Benzoni-Gavage, D. Serre and K. Zumbrun, Alternate Evans functions and viscous shock waves. SIAM J. Math. Anal. 32 (2001) 929-962.

[3] M. Bultelle, M. Grassin and D. Serre, Unstable Godunov discrete profiles for steady shock waves. SIAM J. Numer. Anal. 35 (1998) 2272-2297.

[4] C. Chainais-Hillairet and E. Grenier, Numerical boundary layers for hyperbolic systems in 1-D. ESAIM: M2AN 35 (2001) 91-106.

[5] C. Dafermos, Hyperbolic conservation laws in continuum physics. Springer (2000).

[6] R. A. Gardner and K. Zumbrun, The gap lemma and geometric criteria for instability of viscous shock profiles. Comm. Pure Appl. Math. 51 (1998) 797-855.

[7] M. Gisclon and D. Serre, Étude des conditions aux limites pour un système strictement hyberbolique via l'approximation parabolique. C.R. Acad. Sci. Paris Sér. I Math. 319 (1994) 377-382.

[8] M. Gisclon and D. Serre, Conditions aux limites pour un système strictement hyperbolique fournies par le schéma de Godunov. RAIRO Modél. Math. Anal. Numér. 31 (1997) 359-380.

[9] P. Godillon, Necessary condition of spectral stability for a stationary Lax-Wendroff shock profile. Preprint UMPA, ENS Lyon, 295 (2001)

[10] P. Godillon, Linear stability of shock profiles for systems of conservation laws with semi-linear relaxation. Phys. D 148 (2001) 289-316.

[11] E. Grenier and O. Guès, Boundary layers for viscous perturbations of non-characteristic quasilinear hyperbolic problems. $J$. Differential Equations (1998).

[12] E. Grenier and F. Rousset, Stability of one-dimensional boundary layers by using Green's functions. Comm. Pure Appl. Math. 54 (2001) 1343-1385.

[13] G. Jennings, Discrete shocks. Comm. Pure Appl. Math. 27 (1974) 25-37.

[14] C.K.R.T. Jones, Stability of the travelling wave solution of the FitzHugh-Nagumo system. Trans. Amer. Math. Soc. 286 (1984) 431-469.

[15] T. Kato, Perturbation theory for linear operators. Springer-Verlag (1985).

[16] T.-P. Liu, On the viscosity criterion for hyperbolic conservation laws, in Viscous profiles and numerical methods for shock waves (Raleigh, NC, 1990), pp. 105-114. SIAM, Philadelphia, PA (1991).

[17] T.-P. Liu and Z. Xin, Overcompressive shock waves, in Nonlinear evolution equations that change type. Springer-Verlag, New York, IMA Vol. Math. Appl. 27 (1990) 139-145.

[18] T.-P. Liu and S.-H. Yu, Continuum shock profiles for discrete conservation laws. I. Construction. Comm. Pure Appl. Math. 52 (1999) 85-127.

[19] T.-P. Liu and S.-H. Yu, Continuum shock profiles for discrete conservation laws. II. Stability. Comm. Pure Appl. Math. 52 (1999) 1047-1073.

[20] A. Majda and J. Ralston, Discrete shock profiles for systems of conservation laws. Comm. Pure Appl. Math. 32 (1979) 445-482.

[21] C. Mascia and K. Zumbrun, Pointwise green's function bounds and stability of relaxation shocks. Indiana Univ. Math. J. 51 (2002) 773-904.

[22] D. Michelson, Discrete shocks for difference approximations to systems of conservation laws. Adv. in Appl. Math. 5 (1984) $433-469$.

[23] S. Schecter and M. Shearer, Transversality for undercompressive shocks in Riemann problems, in Viscous profiles and numerical methods for shock waves (Raleigh, NC, 1990), pp. 142-154. SIAM, Philadelphia, PA (1991).

[24] D. Serre, Remarks about the discrete profiles of shock waves. Mat. Contemp. 11 (1996) 153-170. Fourth Workshop on Partial Differential Equations, Part II (Rio de Janeiro, 1995).

[25] D. Serre, Discrete shock profiles and their stability, in Hyperbolic problems: theory, numerics, applications, Vol. II (Zürich, 1998), pp. 843-853. Birkhäuser, Basel (1999).

[26] D. Serre, Systems of conservation laws. 1. Cambridge University Press, Cambridge (1999). Hyperbolicity, entropies, shock waves. Translated from the 1996 French original by I.N. Sneddon.

[27] K. Zumbrun and P. Howard, Pointwise semigroup methods and stability of viscous shock waves. Indiana Univ. Math. J. 47 (1998) 741-871.

[28] K. Zumbrun and D. Serre, Viscous and inviscid stability of multidimensional planar shock fronts. Indiana Univ. Math. J. 48 (1999) 937-992. 